\title{
Software tools for conducting bibliometric analysis in science: An up- to-date review
}

\author{
José A. Moral-Muñoz; Enrique Herrera-Viedma; Antonio Santisteban-Espejo; \\ Manuel J. Cobo
}

How to cite this article:

Moral-Muñoz, José A.; Herrera-Viedma, Enrique; Santisteban-Espejo, Antonio; Cobo, Manuel J. (2020). "Software tools for conducting bibliometric analysis in science: An up-to-date review". El profesional de la información, v. 29, n. 1, e290103.

https://doi.org/10.3145/epi.2020.ene.03

Manuscript received on December, $3^{\text {th }} 2019$ Accepted on December, $15^{\text {th }} 2019$

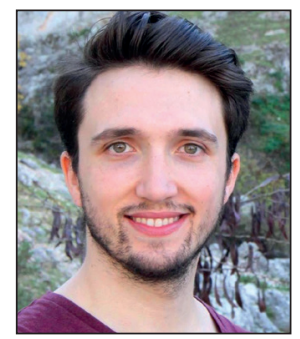

José A. Moral-Muñoz

https://orcid.org/0000-0002-6465-982X

University of Cadiz

Institute of Research and Innovation in

Biomedical Sciences of the Province of

Cadiz (INiBICA)

Cadiz, Spain

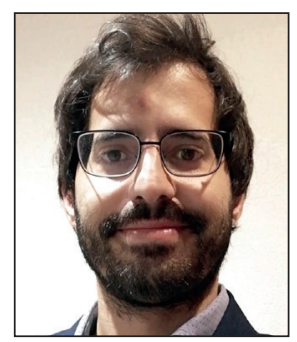

\begin{abstract}
Puerta del Mar Hospital
Division of Hematology and

Hemotherapy

Cadiz, Spain

antonio.santisteban.sspa@

juntadeandalucia.es
\end{abstract}

Antonio Santisteban-Espejo

https://orcid.org/0000-0002-0827-4263 joseantonio.moral@uca.es

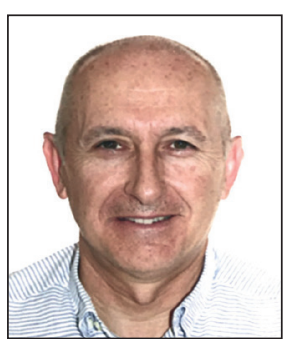

Enrique Herrera-Viedma

https://orcid.org/0000-0002-7922-4984

University of Granada

E.T.S. de Ingeniería Informática y de

Telecomunicación

Periodista Daniel Saucedo Aranda, s/n.

18014 Granada, Spain

viedma@decsai.ugr.es

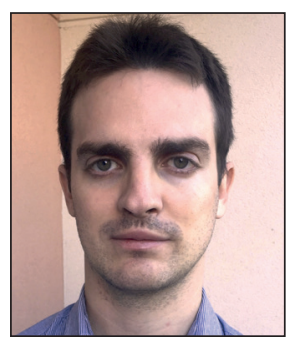

\begin{abstract}
Bibliometrics has become an essential tool for assessing and analyzing the output of scientists, cooperation between universities, the effect of state-owned science funding on national research and development performance and educational efficiency, among other applications. Therefore, professionals and scientists need a range of theoretical and practical tools to measure experimental data. This review aims to provide an up-to-date review of the various tools available for conducting bibliometric and scientometric analyses, including the sources of data acquisition, performance analysis and visualization tools. The included tools were divided into three categories: general bibliometric and performance analysis, science mapping analysis, and libraries; a description of all of them is provided. A comparative analysis of the database sources support, pre-processing capabilities, analysis and visualization options were also provided in order to facilitate its understanding. Although there are numerous bibliometric databases to obtain data for bibliometric and scientometric analysis, they have been developed for a different purpose. The number of exportable records is between 500 and 50,000 and the coverage of the different science fields is unequal in each database. Concerning the analyzed tools, Bibliometrix contains the more extensive set of techniques and suitable for practitioners through Biblioshiny. VOSviewer has a fantastic visualization and is capable of loading and exporting information from many sources. SciMAT is the tool with a powerful pre-processing and export capability. In views of the variability of features, the users need to decide the desired analysis output and chose the option that better fits into their aims.
\end{abstract}

\section{Keywords}

Bibliometrics; Scientometrics; Science mapping analysis; Tools; Bibliographic databases; Performance analysis; Software; Libraries; $R$-package; Python-package; Software review.

\section{Financing}

This article has been possible thanks to Feder funds (TIN2016-75850-R). 


\section{Introduction}

Science can be defined as a social activity oriented to characterize a field of knowledge through observation and measurement, being performed by research communities and influenced by societal conditions (communication media, Government policies and legal system). In accordance with Asimov (2010), modern science emerged when nature was considered under the watchful eye of measurement methods. Thus, professional and researchers require a body of theoretical and practical tools to quantify experimental

Researchers require a body of theoretical and practical tools to quantify experimental data

data. In this sense, Van-Raan (2004a) also discussed this topic when recorded the sentence attributed to Onnes: "Measuring is knowing". To measure is, then, the basis for the building of science. However, how science itself can be measured?

This view of the matter presides the methodical foundations of bibliometrics, that is, the quantitative study of production, growth, maturation and consumption of scientific publications. In this sense, the term "bibliometrics" was originaIly coined by Pritchard (1969), replacing so the classical "statistical bibliographies". Since then, bibliometrics has quickly evolved and technically perfected alongside with the exponential growth of science. Presently, the massive amount of data published on academic journals, books, patents, proceedings, etc. required to be stored and organized into bibliographic databases. The information contained on these platforms (i.e. citations, keywords, titles, journals, authors, institutions, etc.) provides a valuable sample to perform science evaluation research using bibliometric techniques (Gutiérrez-Salcedo; Martínez; Moral-Muñoz; Herrera-Viedma; Cobo, 2018).

As a result, bibliometrics has become in contemporary context an essential tool for assessing and analyzing researcher's production (Ellegaard; Wallin, 2015), collaboration between institutions (Skute; Zalewska-Kurek; Hatak; de Weerd-Nederhof, 2019), impact of state scientific investment in national R\&D productivity (Fabregat-Aibar; Barberà-Mariné; Terceño; Pié, 2019) and academic quality (Van-Raan, 1999), among other possibilities (Glänzel, 2012). Concerning to bibliometrics, it can be divided in two major fields of study or subject areas: performance analysis and science mapping analysis (SMA) (Noyons; Moed; Van-Raan, 1999; Cobo; López-Herrera; Herrera-Viedma; Herrera, 2011a). On the one hand, performance analysis aims to evaluate different scientific actors (researchers, institutions, countries, etc.) through bibliographic indexes based on publications and citations data (Narin; Hamilton, 1996). On the other hand, SMA lies on the topological and temporal representation of the cognitive and social structure of a particular research field (Small, 1999; Cobo; López-Herrera; Herrera-Viedma; Herrera, 2011).

Concerning the current published documents, there are several reviews about the tools available to perform bibliometric analyses. Moral-Muñoz, López-Herrera, Herrera-Viedma \& Cobo (2019) reviewed the freely available SMA software and allowing to perform the complete analysis, without using external software to preprocess or visualize the analysis. Pan, Yan, Cui \& Hua, (2018) evaluated how the three SMA software (CiteSpace, HistCite and VOSviewer) is used, cited and diffused. Gutiérrez-Salcedo, Martínez, Moral-Muñoz, Herrera-Viedma \& Cobo, 2018) introduced the essential techniques and software tools to analyze the impact of a research field and its scientific structure. Chen (2006; 2017; 2019) introduced CiteSpace, VOSviewer and CitNetExplorer to perform an applied analysis using SMA. Pradhan (2016) described some of the commonly used software tools to perform SMA analysis. Sangam \& Mogali (2012) highlight the different capabilities of mapping and visualization software tools. Finally, Cobo,López-Herrera, Herrera-Viedma \& Herrera (2011b) performed an in-depth analysis of the advantages, drawbacks and most important differenced among the SMA software tools. Nevertheless, those reviews are conditioned for temporal factors. These tools have been improved, and new features have been incorporated; furthermore, other described tools are no longer maintained, or there are powerful new options.

In this sense, the present review aims to offer an up-to-date review of the different tools available to perform bibliometric and scientometric studies; including the data acquisition sources, performance analysis, science mapping and visualization tools. For this purpose, the structure of the study is divided into three sections and organized as follows: Section 2) describes the method and selection criteria. Section 3) shows the different databases from which to get the information. Section 4) presents general bibliometric and performance analysis tools. Section 5) analyzes the main SMA software tools. Section 6) describes and analyzes the available libraries. Finally, in Section 7) some discussion and concluding remarks are highlighted.

\section{Methods - Selection criteria}

In order to provide an adequate state-of-art of the available tools to perform bibliometric and scientometric analyses, some inclusion criteria have been previously established:

- Database that allows downloading bibliographic data.

- General bibliometric software, or based on indexes and performance analysis.

- SMA tools.

- $R$ and Python libraries. 
All of these software and tools have to be in a final form and with an evident up-to-date status. Furthermore, we only include those that are focused explicitly on the bibliometrics, not general tools that could be employed in some of the processing stages.

\section{Bibliometrics has become an essential tool for assessing and analyzing the pro- ductivity and impact of academics and technology}

\section{Databases}

In order to perform a bibliometric analysis, the first stage is to decide the best data source that fits with the scientific coverage of our research area. It is important to highlight that the number of bibliographic databases is high (e.g. PubMed, EMbase, SpringerLink, etc.), but not all of them provide information that allows easily performing bibliometric analyses. Thus, the present document shows the main bibliographic databases used in bibliometrics analysis: Web of Science (WoS), Scopus, Google Scholar (GS), Microsoft Academic (MA) and Dimensions. In what follows, a short description of each database is shown, and also, the main characteristics focusing on the bibliometric analyses are shown in Table 1.

Table 1. Characteristics of the databases

\begin{tabular}{|l|l|l|l|l|l|}
\hline Database & Subscription & Data download & Records limit & API \\
\hline WoS & Yes & Yes & $\begin{array}{l}500 \text { records with a total of } \\
100,000 \text { per query }\end{array}$ & Yes & Plain text and tab-delimited \\
\hline Scopus & Yes & Yes & Fo & n/a & Yes \\
\hline GS & Free & Using the API & n/a & RIS and CSV \\
\hline MA & Free & 50,000 & N/a & Yes \\
\hline Dimensions & Free or under subscription & Yes & & Yes & CSV or Excel \\
\hline
\end{tabular}

- Web of Science: It is a website that provides access to multiple databases and citation data for 256 disciplines (science, social science, arts and humanities). The access is under subscription. The Institute for Scientific Information (ISI) was the original producer, after that, its intellectual property passed to Thomson Reuters, and now the maintenance is in charge of Clarivate Analytics. It covers different formats, such as full-text articles, reviews, editorials, chronologies, abstracts, proceedings (journals and book-based) and technical papers. The total number of records is beyond 90 million. Its temporal coverage is from 1900 to the present.

- Scopus: It is a website that offers access to databases and citation data in life sciences, social sciences, physical sciences and health sciences. Elsevier provides access to Scopus, and a subscription is needed. It covers three types of sources: book series, journals and trade journals. Furthermore, the searches performed in Scopus also incorporate searches in patent databases. The number of records is around 69 million. The temporal coverage is from 2004 to present.

- Google Scholar: It is a freely available website, launched in 2004, that indexes the full text or metadata of the scientific literature from the most peer-reviewed online academic journals, books, conference papers,

The first stage is to decide the best data source that fits with our research area theses, preprints, abstracts, technical reports, court opinions and patents. Google does not provide the number of records, but (Gusenbauer, 2019) established an estimation of 389 million documents in 2018. It was launched in 2004. GS has been criticized for not ban the predatory journals (Ibba; Pani; Stockton; Barabino; Marchesi; Tigano, 2017; Chapman; Ellinger, 2019).

- Microsoft Academic: It was previously called as Microsoft Academic Search, but it was relaunched as a new service in 2016, as MA. The supply and maintenance are in charge of Microsoft. It is offered as a free public web search engine. According to the information available on the webpage, it currently indexes over 230 million publications, 88 million are journal articles. The first studies suggest that it is a competitor of the main databases, GS, WoS and Scopus (Harzing; Alakangas, 2017; Hug; Ochsner; Brändle, 2017; Haunschild; Hug; Brändle; Bornmann, 2018).

- Dimensions: It is a new database that, according to its available information, its goal is to provide "a more open and comprehensive data infrastructure that empowered users to explore connections between a wide range of research data". It is supported by Digital Science \& Research Solutions Inc. and has been considered as an alternative to WoS and Scopus (Orduña-Malea; Delgado-López-Cózar, 2018; Thelwall, 2018). Different products are available, with a free version option. It offers access to the traditional document types (articles, conference papers, books, etc.), but also includes links to grants, patents, clinical trials, datasets and policy papers. The number of records that contain Dimensions is higher than 102 million publications in 2019. It was launched in 2018. They follow the Open Access movement, arguing that the producers of the data should not develop the metrics.

\section{Software tools for conducting performance bibliometric analysis}

Once the data is obtained, the analyst could develop a performance analysis process. In this process, information about the production and impact of a specific research area is obtained. Several indicators could be extracted from the publi- 
cation core, such as number of publications, number of citations, highly cited publications, number of non-cited publications, research field classification and normalized citations. In that way, the software tools could offer the indicators that the developer considers adequate to the scientific evaluation.

Recently, alternative metrics, also known as Altmetrics, have been developed to evaluate scientific impact at social media (Bornmann; Haunschild, 2018). This approach describes a web-based metric for the impact of publications and other scholarly material by using data from social media platforms (i.e. Twitter, Facebook, Google+, blogs, Mendeley, CiteULike, Reddit and Wikipedia, among others) (Veeranjaneyulu, 2017). The appearance of this type of measures is related to the social media revolution; there are now different groups of the population, non-author professionals, which read research articles and now also share them, and new types of academic outputs have appeared (Bornmann; Williams, 2013). Nonetheless, this kind of indicators is still under development and are not integrated into the existing software tools. Probably, these new metrics will be included in future software tools.

Thus, under this category, the most relevant software tools that still available have been included in this review. We should mention that in the past there were other important software tools focused on bibliometric performance analysis, but currently are unmaintained, such for example, the software HisCite which was used to visualize the results of the searches performed in the WoS, allowing to analyze and organize the results to obtain the topic's structure, history and relationships. Nevertheless, it was not included in the present reviews since Clarivate informed about no longer maintained.

In what follows, the description and main characteristics of these software tools are shown. Also, Table 1 shows the main details. In this software tool category, we will focus on the data sources used, the kind of indicators and analysis available.

Table 2. Characteristics of the performance software

\begin{tabular}{|l|l|l|l|l|l|l|}
\hline \multicolumn{1}{|c|}{ Software } & $\begin{array}{l}\text { Analyzed } \\
\text { version }\end{array}$ & \multicolumn{1}{|c|}{ Year } & \multicolumn{1}{c|}{ Developer } & Operative System & \multicolumn{1}{c|}{ Data source } \\
\hline CRExplorer & 1.9 & 2018 & $\begin{array}{l}\text { Hochschule für Telekommu- } \\
\text { nikation Leipzig (HfTL) }\end{array}$ & Java & $\begin{array}{l}\text { WoS, Scopus and } \\
\text { Crossref }\end{array}$ \\
\hline Publish or Perish & 7 & 2019 & $\begin{array}{l}\text { Melbourne-based Tarma } \\
\text { Research Software Pty Ltd. }\end{array}$ & Win, Mac and Linux & $\begin{array}{l}\text { WoS, Scopus, GS, } \\
\text { Crossref, MA }\end{array}$ & Desktop \\
\hline ScientoPyUl & 1.4 .0 & 2019 & University of Cauca & Python & WoS and Scopus & Web \\
\hline
\end{tabular}

The descriptions of the main performance analysis tools are here below:

- CRExplorer: It is indicated to the disambiguation and analysis of the cited references of a publication collection downloaded from WoS database, Scopus or Crossref (Thor; Marx; Leydesdorff; Bornmann, 2016). It was developed for applying the Reference Publication Year Spectroscopy (RPYS), which allows identifying the publications with most sig-

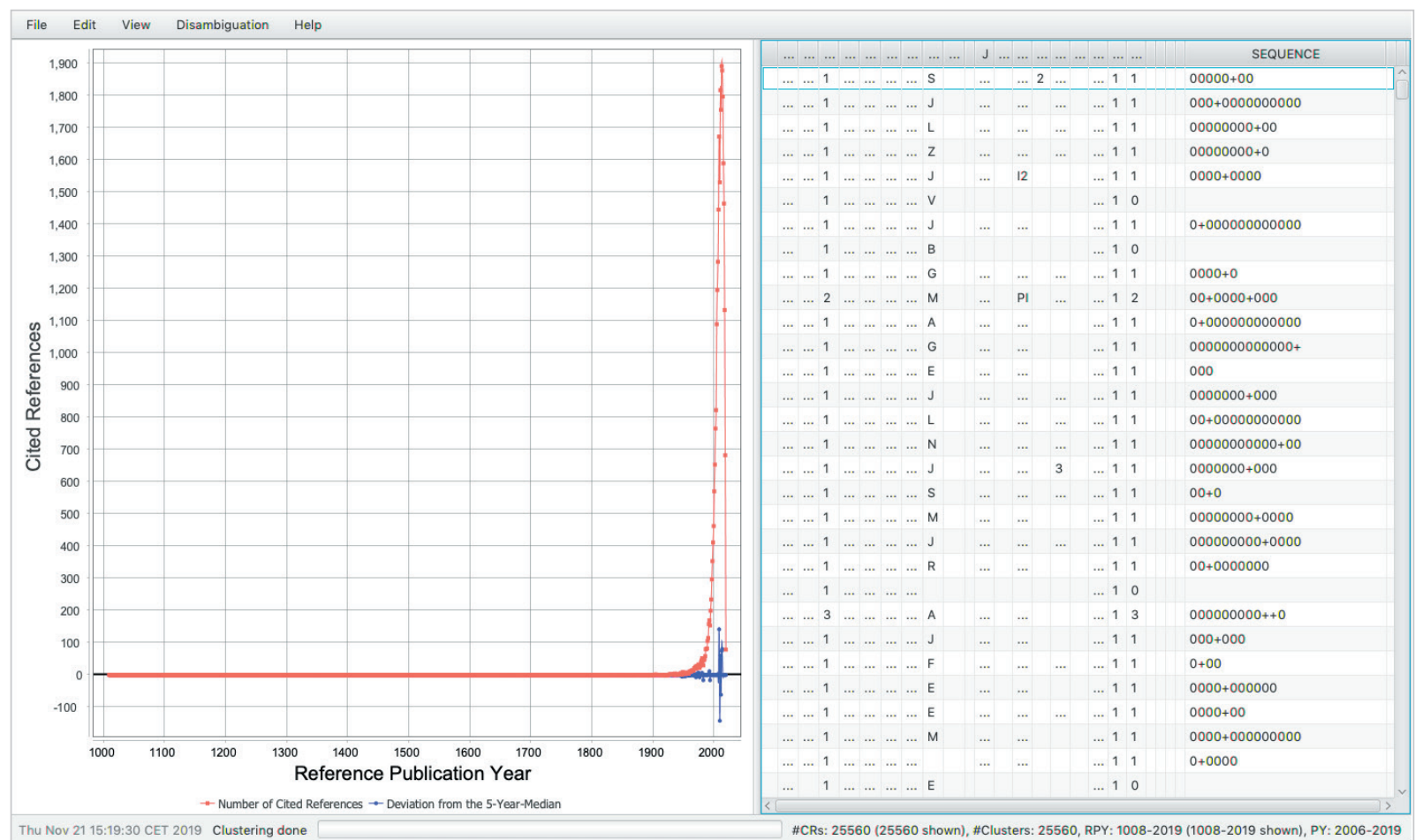

Figure 1. Analysis of the EPI journal with CRExplorer 
nificant influence in a certain research area, topic, or in general, a set of documents. It is based on

"the analysis of the frequency with which references are cited in the publications of a specific research field in terms of the publications years of these cited references" (Marx; Bornmann; Barth; Leydesdorff, 2014).

In short, CRExplorer shows the spectrogram of a topic, cleans the cited references (disambiguation) and uses a smoothing algorithm to correct the noise. Due to its development using Java programming, it runs on most hardware and operating system platforms. Furthermore, CRExplorer offers two options to run an analysis: Java Web Start and download a runnable JAR file. An interesting feature is the analysis of sequences over time, where the citations are considered on average, above average and below average for each year; it allows to identify sleeping beauty (Van-Raan, 2004b), constant performer, hot paper and life cycle publications. Data output could be exported to WoS, Scopus or CSV (graph, cited references and/or citing publications).

- Publish or Perish: It was primarily developed to generate the h-index from GS search results and was Anne-Wil Harzing who initiated and guided the development of this software (Harzing, 2008; Harzing; Van-der-Wal, 2008). An interesting feature is that we can perform searches directly to GS, WoS, Scopus, Crossref and MA. Furthermore, it allows to import external data instead of performing the search. It provides a performance analysis of the core of documents obtained from the indicated source. In that sense, it interesting to highlight the possibility of obtaining the h-index average annual increase, age-weighted citation rate and authors per paper indicators. Furthermore, the output can be exported to BibText, CSV, EndNote, ISI Export, JSON Export and RefMan/RIS; it is interesting to perform further analyses in other tools, although it is limited to 1000 records.

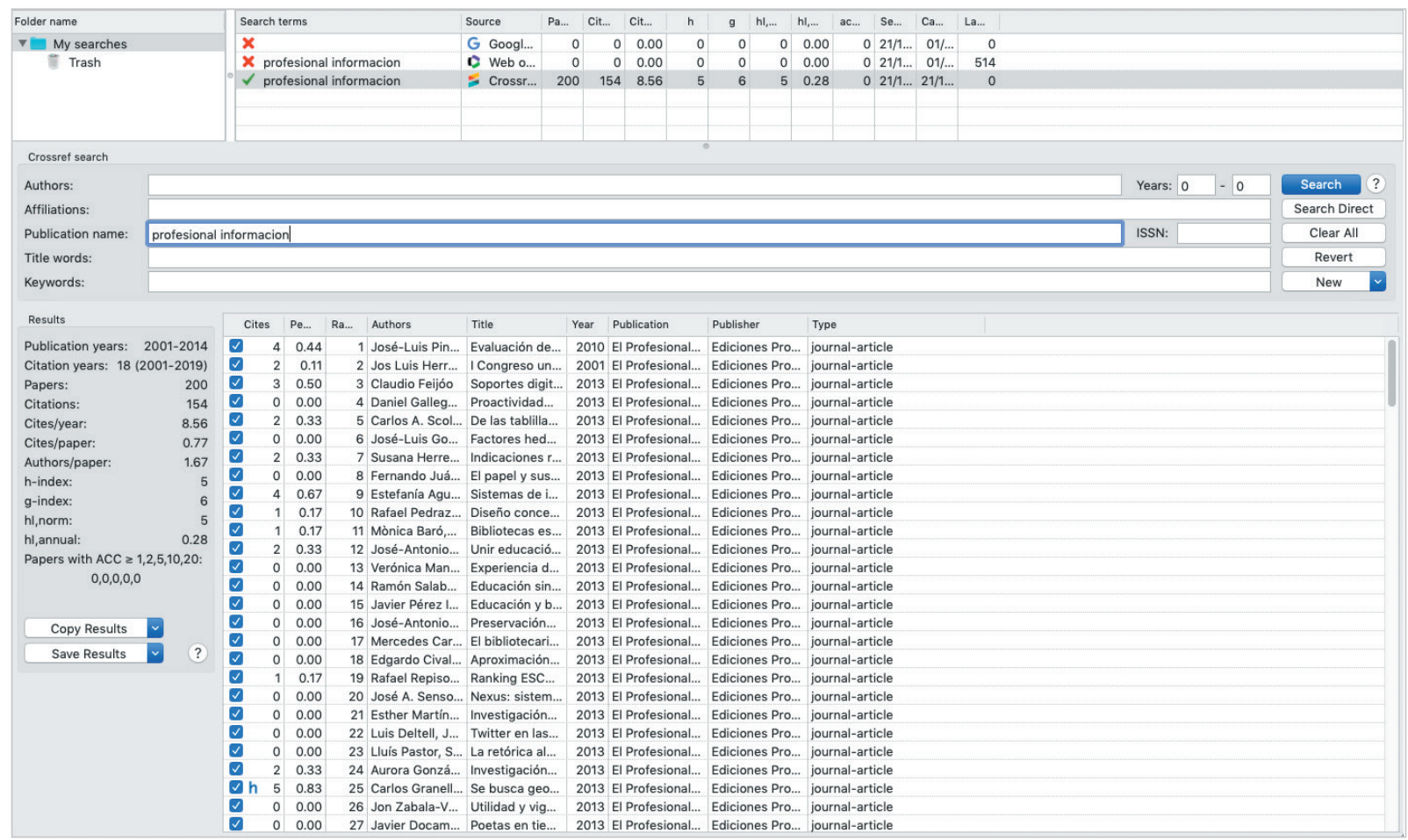

Figure 2. Analysis of the EPI journal with Publish or Perish

- ScientoPyUI: This is not a complete software, and it is needed to run it under Python. It is the graphical user interface (GUI) for the ScientoPy (described in Section 6) (Ruiz-Rosero; Ramírez-González; Viveros-Delgado, 2019). Although the characteristics of ScientoPy will be described below, some comments will be addressed. Thus, we can perform a preprocessing stage, consisting on document type filtering, author's name normalization, duplicate removal, times cited and document's country and institutions. Concerning the analysis options, it allows performing top and specific topics evaluation and wildcard search. Furthermore, ScientoPy adds some performance indicators to the topics analyzed, such as average growth rate, average document per years and percentage of documents in There are substantial differences among the exporting options of the databases the last years. Although this tool does not provide sophisticated maps, the visualization options are: timeline, horizontal bars, horizontal bars trends, evolution and word cloud. Graphs can be exported to EPS, SVG and PNG. 


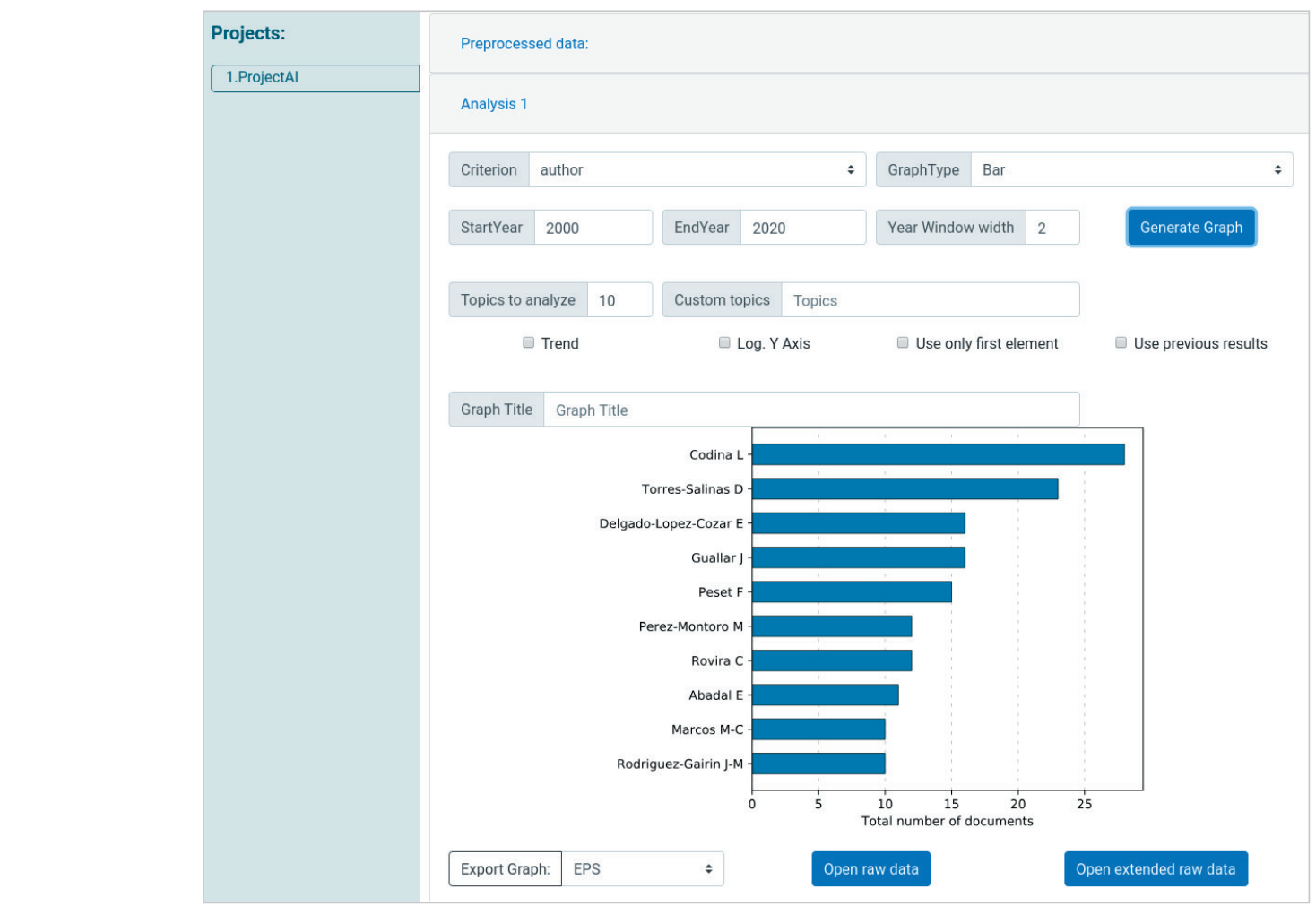

Figure 3. Analysis of the EPI journal with ScientoPyUI

\section{Software tools for conducting science mapping bibliometric analysis}

The bibliometrics analysis could be enhanced with scientific maps representing the relationship among the different actors (authors, institutions, countries, etc.). A map of science is, therefore, a spatial representation of how disciplines, fields, specialties and individual papers or authors are related to one another as shown by their physical proximity and relative locations (Small, 1999). In the last years, several reviews have analyzed the most relevant software (Cobo; López-Herrera; Herrera-Viedma; Herrera, 2011; Sangam; Mogali, 2012; Pradhan, 2016; Chen, 2017), nonetheless, they change over time, and some new appeared. Therefore, some updated characteristics of the main SMA software are shown in Table 3:

Table 3. Characteristics of the SMA tools

\begin{tabular}{|c|c|c|c|c|c|}
\hline Tools & Analyzed version & Year & Developer & Operative System & User interface \\
\hline Bibexcel & 2017 & 2017 & University of Umeå (Sweden) & Win & Desktop \\
\hline Biblioshiny & & 2019 & University of Naples Federico II (Italy) & Runs in $R$ & Web \\
\hline BiblioMaps & 3.2 & 2018 & University of Lyon (France) & Runs in Python & Web \\
\hline CiteSpace & 5.5.R2 & 2019 & Drexel University (USA) & Win & Desktop \\
\hline CitNetExplorer & 1.0 .0 & 2014 & Leiden University (The Netherlands) & Win, OSX, Linux & Desktop \\
\hline SCIMAT & 1.1 .04 & 2016 & University of Granada (Spain) & Win, OSX, Linux & Desktop \\
\hline Scir Tool & 1.3 & 2018 & $\begin{array}{l}\text { Cyberinfrastructure for Network Science } \\
\text { Center (USA) }\end{array}$ & Win, OSX, Linux & Desktop \\
\hline VOSviewer & 1.6.13 & 2019 & Leiden University (The Netherlands) & Win, OSX, Linux & Desktop \\
\hline
\end{tabular}

In what follows, a summarized description of the main characteristics of each software tool is provided. Moreover, for each one, a small bibliometric analysis has been developed using a set of articles from EPI journal, showing an example of screenshot.

- Bibexcel: It is intended to create data files that can be imported to Excel, or any program that takes tabbed data records for further handling (Persson; Danell; Wiborg Schneider, 2009). It was developed by Olle Persson at University of Umeä (Sweden). This software incorporates various tools, some of them visible in the window and others hole up behind the menus. The main characteristic of this software is the flexibility, but for this reason, it could be initially perceived as challenging to use by new users. BibExcel can read information retrieved from various bibliographic sources, for example, WoS, Scopus, and the ProCite export format. Nevertheless, if the user learns the typical Bibexcel file structure, different types of document can be formatted in order to be analyzed. Different bibliometric networks 


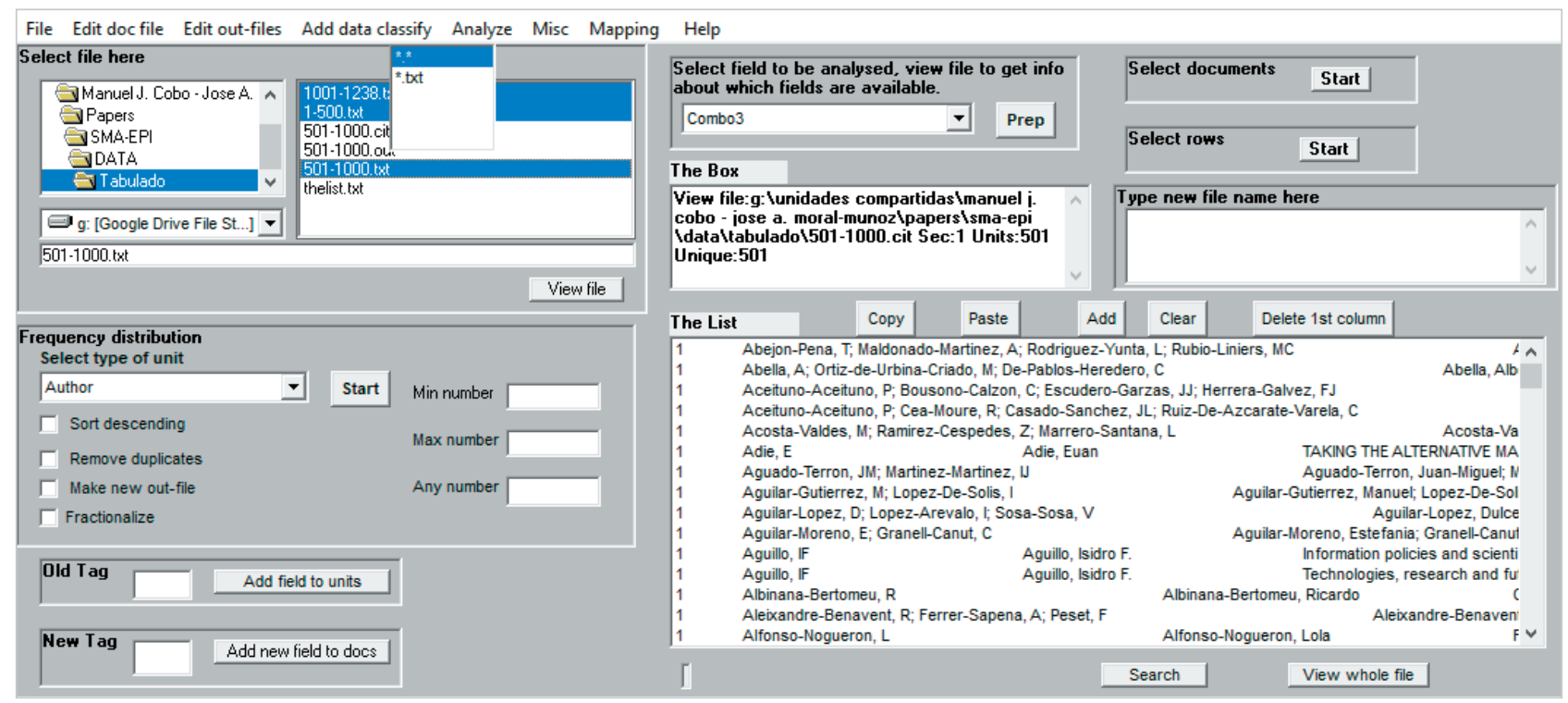

Figure 4. Analysis of the $E P I$ journal with Bibexcel

can be obtained using the parameters offered by this tool. The main networks are: co-citation, bibliographic coupling, co-author, and co-word. Moreover, it is possible to create different co-occurrence matrices taking any document's field, or combining some of them. Once the data is normalized, the user can apply a clustering algorithm or prepare a matrix to perform a Multidimensional Scaling (MDS), using an external tool. On the other hand, it allows submitting the textual data to different preprocessing tasks, such as an English word stemmer, documents deduplication and text transformation (keep author's first initial, convert comma-delimited addresses, etc.). Besides, Bibexcel empowers the deletion of low recurrence items and keeps just the strongest links. Although it has not visualization option, it incorporates different export options that allow visualizing the data using external software like Pajek (Batagelj; Mrvar, 2004), Ucinet (Borgatti; Everett; Freeman, 2002), SPSS or VOSviewer (Van-Eck; Waltman, 2010). The type of visualization selection will depend on the nature of the unit of analysis.

- Biblioshiny: Paraphrasing the own tool slogan, Biblioshiny is "bibliometrix for no coders". It is powered by Bibliometrix (Aria; Cuccurullo, 2017) and is its web-based graphical interface. Although Bibliometrix will be described below, it was programmed in the $R$ language in order to facilitate the interconnection with other $R$ packages. It was developed by Massimo Aria and Corrado Cuccurullo from the University of Naples and University of Campania's Luigi Vanvitelli (Italy). It mainly works with WoS, Scopus and Dimensions data. The interface is intuitive and well organized, and the
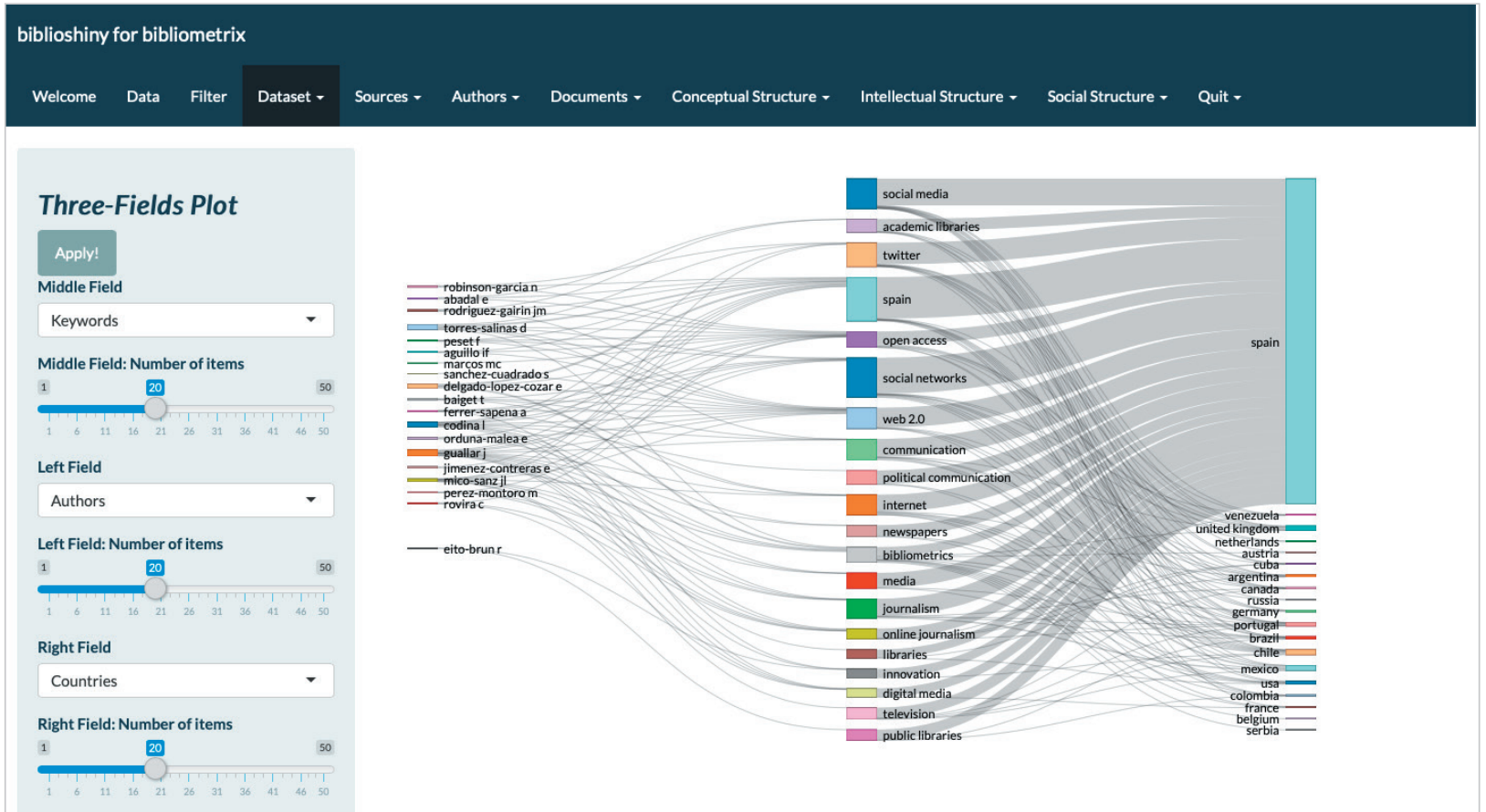

Figure 5. Analysis of the EPI journal with Biblioshiny 


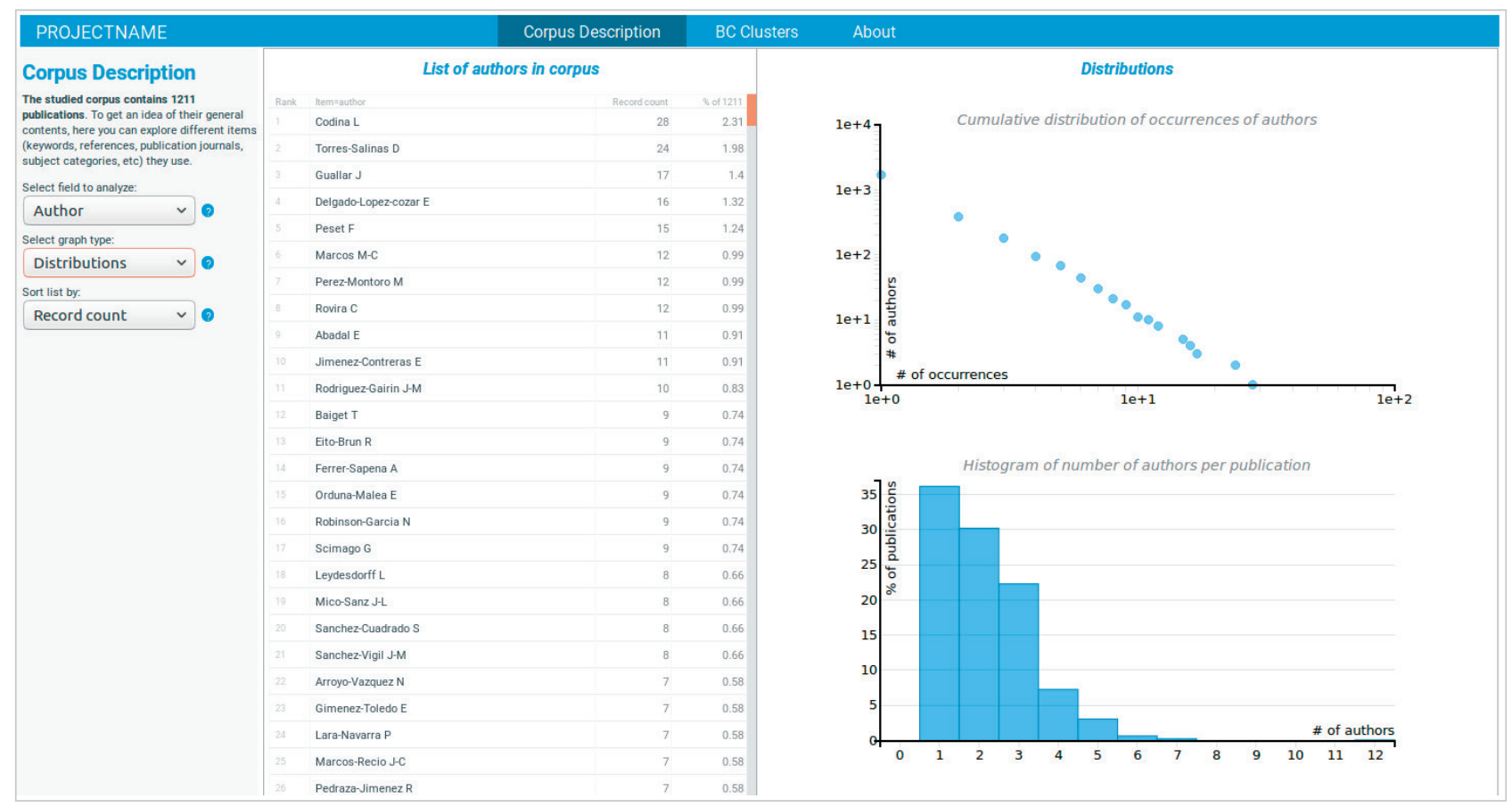

Figure 6. Analysis of the EPI journal with BiblioMaps

developers have divided the main menu according to the SMA workflow. This menu incorporates analytics and graphs for three-level metrics (source, author and document) and three structures of knowledge (conceptual, intellectual and social). The analysis options are diverse and are subdivided into 7 categories, divided in the analytics and graphs stated before: 1) Overview, 2) Sources, 3) Authors, 4) Documents, 5) Conceptual structures, 6) Intellectual structure, and 7) Social structure. The graphs and performance analyses generated can be exported to several kinds of file formats; maps can be exported to Pajek and html, and tables can be copied to the clipboard or saved as Excel, pdf or printed.

- BiblioMaps: BiblioTools is a set of scripts to perform SMA based on bibliographic data (Grauwin; Jensen, 2011). Although these scripts run under Python, in an update in 2016-2017, the author, Sébastian Grauwin developed the web interface so-called BiblioMaps. This software is designed to not require knowledge about Python, but BiblioMaps makes this issue more manageable. Furthermore, the user can change the code to get different outputs. It can work with WoS and Scopus data. This set of scripts perform the following tasks: i) Data acquisition, ii) Data preprocessing, applying to parse and filtering to the raw data, iii) Data analysis, obtaining co-occurrence networks, bibliographic coupling and clusters, iv) Data visuali-

\section{Almost all software tools and libraries can import data downloaded from WoS and Scopus}

exported to other software, and v) Data report, the analysis obtained can be exported to LaTeX format. BiblioMaps only offer the network as a visualization option, to obtain different maps external software is needed. Although the user can perform the analysis controlling each stage of the process, this tool offers the "all_in_one" script, allowing to perform all the analyses at once.

- CiteSpace: It allows the analysis and visualization of trends and patterns in a research area (Chen, 2006; Chen, 2019). The main goal of this tool is to facilitate the analysis of emerging trends in a knowledge domain. It was developed at Drexel University (USA). This tool offers several options to understand and interpret network and historical patterns, such as the growth of a topic area, the main citations in the knowledge base, the automatic labelling of the different clusters using terms from citing articles, geospatial collaboration network, and international collaboration. CiteSpace is able to work with different bibliographic databases, such as WoS, Scopus, and Chinese Social Science Citation Index (Cssci). Furthermore, it supports citation-based studies using open-access sources, such as Dimensions and Crossref. Other no citation-related platforms can be analyzed with a small set of visual analytics functions, such as PubMed, China National Knowledge Infrastructure (CNKI) and ProQuest Dissertations and Theses. Some other interesting features are incorporated, such as the direct download from Crossref. The CiteSpace workflow is briefly described in the following phases: i) Knowledge domain detection, ii) Data collection, iii) Extract research front terms, iv) Time slicing, v) Threshold selection, vi) Pruning and merging, vii) Visual inspection, and viii) Pivotal points verification. CiteSpace offers several possibilities to visualize and perform analysis from the built networks. The user obtains a visualization where the pivotal points are shown about their betweenness centrality. They are highlighted in the software window 


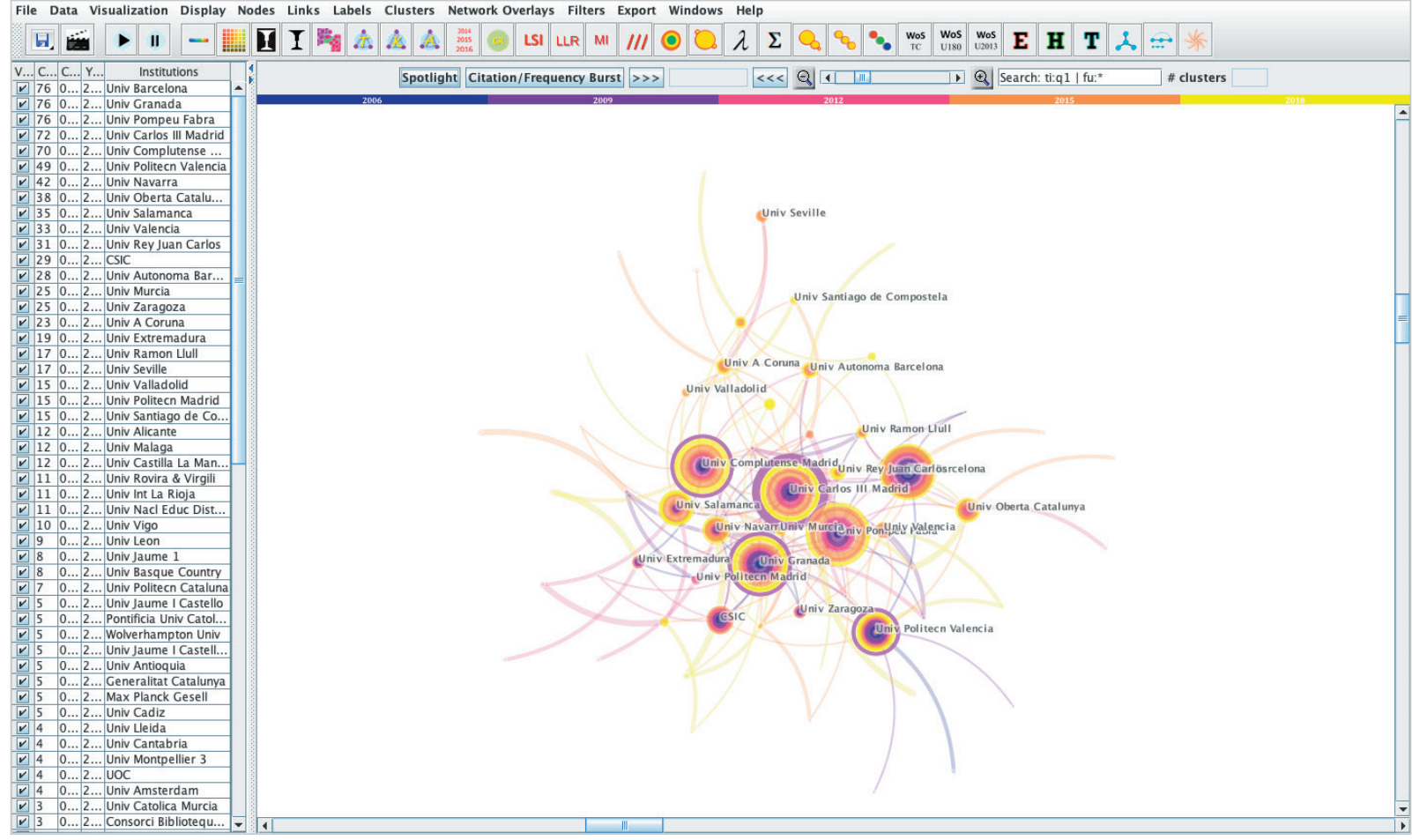

Figure 7. Analysis of the EPI journal with CiteSpace

with a purple ring surrounded by a tree ring. Finally, it is interesting to highlight the geographical visualization option. Authors' geographic locations can be mapped as a geospatial map in KML. To obtain this representation, the Google Earth interface can be used. Then, it is possible to explore the authors' locations and links to their collaborators, and also redirect to the original articles directly within Google Earth.

- CitNetExplorer: It is a software tool for visualizing and analyzing citation networks, based on the algorithmic historiography designed by Garfield (Garfield; Pudovkin; Istomin, 2003). In the networks obtained by CitNetExplorer (Van-Eck; Waltman, 2014), each node represents a publication. Each edge represents a citation relation between two publications. It was developed by the Centre for Science and Technology Studies (CWTS) at Leiden University (The Netherlands). The

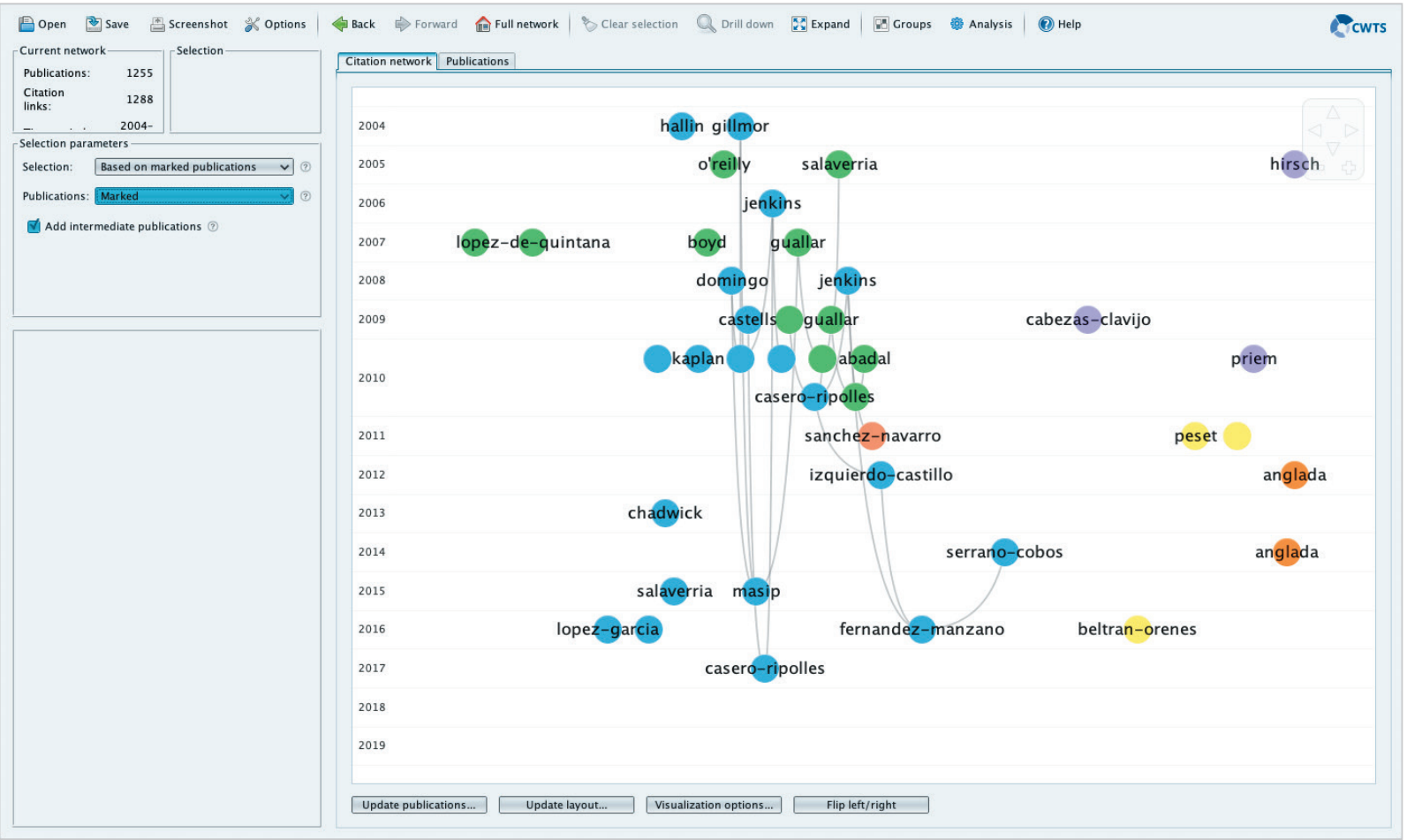

Figure 8. Analysis of the EPI journal with CitNetExplorer 
information to construct citation networks is collected uniquely from WoS data. Nevertheless, it is not restricted to this database; Scopus data could be analyzed equally. Once the citation network is formed, it can be exported into Pajek file format (Batagelj; Mrvar, 2004). CitNetExplorer uses two different approaches. One approach consists of providing both data from publications and data from citations relations among publications. The other approach is based on adding the data directly downloaded from WoS. It presents three remarkable functionalities: the capability of select publications, drill-down and expand functionalities and different algorithms to generate the network. Concerning the analysis options offered by CitNetExplorer, four different options are provided: extract connected components, cluster publications, identify core publications and find the shortest or the longest path from a publication to others. Furthermore, not only the direct citation relations are visible, the visualization can be set to show higher-order indirect citation relations. An additional feature is the possibility of launch CitNetExplorer directly from the web page.

- SciMAT: It is an open-source (GPLv3) SMA software tool designed to assist all the steps in SMA workflow, incorporating all the necessary elements (methods, algorithms and measures) to obtain the different analyses and visualizations (Cobo; López-Herrera; Herrera-Viedma; Herrera, 2012). SciMAT was developed by the Secaba Lab at University of Granada (Spain), and it is now updated and maintained by the IntellSOK group at University of Cadiz (Spain). It incorporates all modules to perform all the steps of the SMA workflow. It supports the analyst to carry out all the different steps, from the data loading to the visualization and interpretation of the output. The science maps obtained are enriched with bibliometric measures based on citations, such as the sum, maximum, minimum, and average citations. Moreover, it uses advanced bibliometric indexes such as the h-index (Hirsch, 2005; Alonso; Cabrerizo; Herrera-Viedma; Herrera, 2009), G-index (Egghe, 2006), HG-index (Alonso; Cabrerizo; Herrera-Viedma; Herrera, 2010) and $\mathrm{q}^{2}$-index (Cabrerizo; Alonso; Herrera-Viedma; Herrera, 2010). SciMAT allows users to add files in WoS and RIS formats. Then, it incorporates a preprocessing module where de-duplicating (manual, by plural or by Levenshtein distance, or importing from an XML file), time-slicing, data reduction and network reduction can be performed. SciMAT divides the analysis process into four main stages: i) Dataset building, ii) Creation and normalization of the network, iii) Application of a clustering algorithm to get the map and its associated clusters or subnetworks, and iv) network, performan$c e$, and longitudinal analyses. Different visualization techniques are available in SciMAT, such as a strategic diagram, cluster network, evolution map, and overlapping map. Finally, it is interesting to remark that the visualization module can build a report in html or LaTeX format. The images (strategic diagrams, overlapping-items map, etc.) are exported in PNG and SVG formats so the user can easily edit them. Furthermore, the cluster networks and evolution maps are exported in Pajek format (Batagelj; Mrvar, 2004).

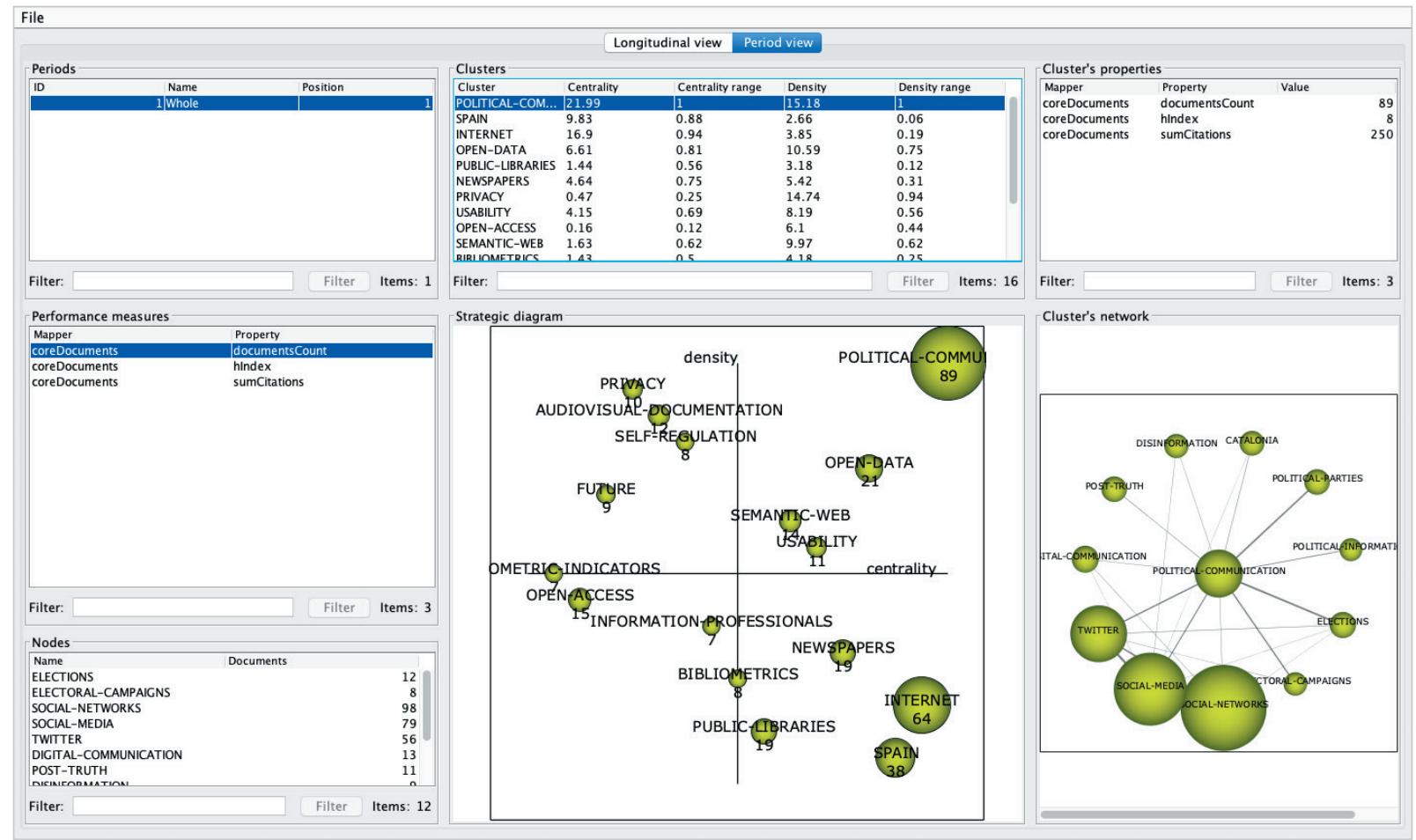

Figure 9. Analysis of the EPI journal with SciMAT 


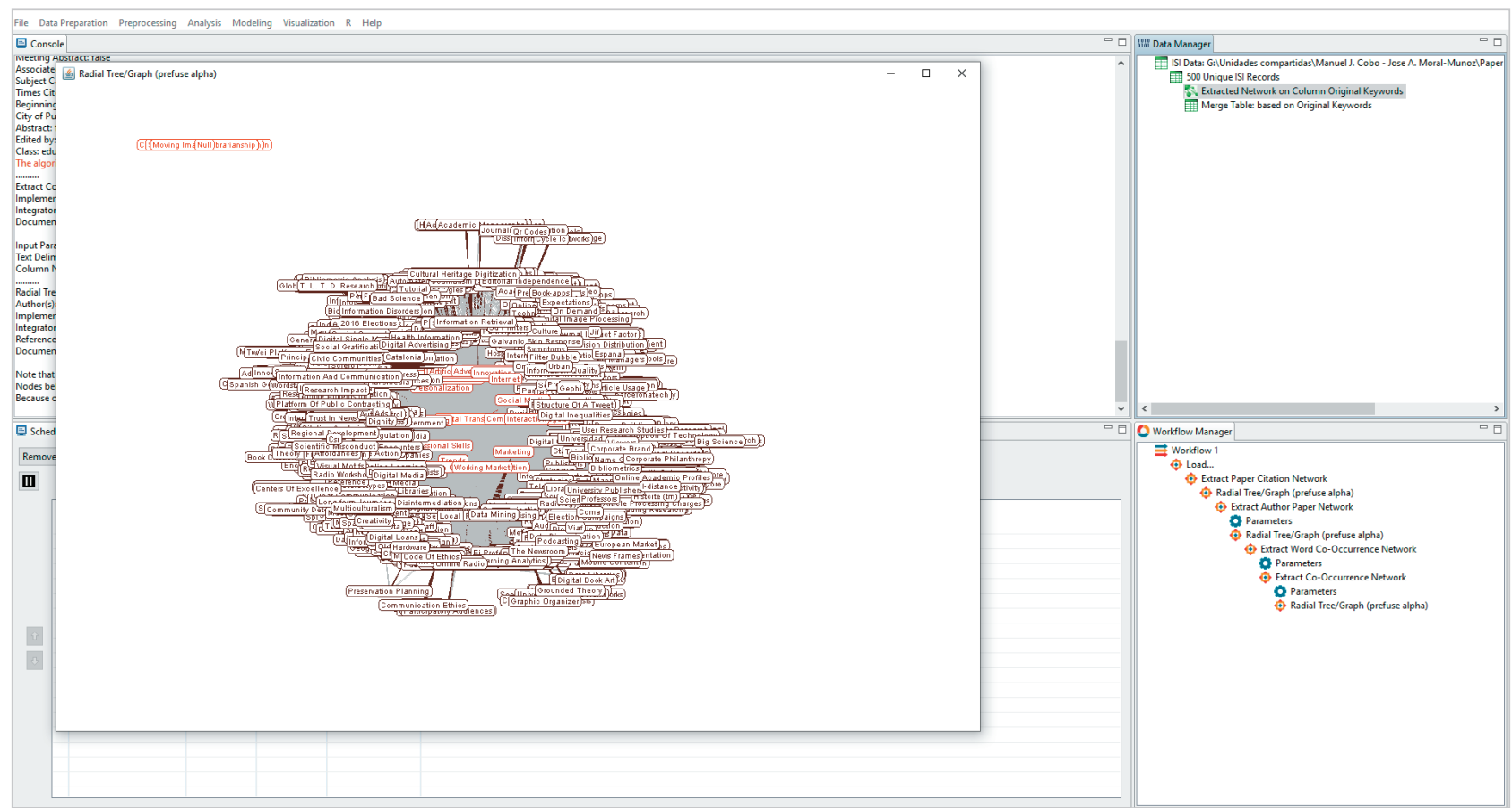

Figure 10. Analysis of the EPI journal with $S c i^{2}$ Tool

- Sci Tool: It is a modular toolset particularly intended to play out the research of science (Sci2 Team, 2009). It supports temporal, geospatial, topical, and network analysis and the representation of datasets at the micro (individual), meso (local), and macro (global) levels. It was developed by Cyberinfrastructure for Network Science Center at Indiana University (USA). It read several bibliographic data formats, such as WoS, Scopus, GS, Bitext and the exportation data format of EndNote. Furthermore, it can analyze data information from social media like Facebook, research funding from the National Science Foundation and National Institutes of Health, as well as other academic data in CSV format. As can be observed, this tool supports a wide variety of information sources. The Sci Tool workflow is based on the typical science study (Börner; Chen; Boyack, 2003): Data acquisition and processing, Data analysis, Modeling, and Layout. It allows extracting different types of networks, performing several analyses (temporal, geospatial, textual and networks). Mainly, this tool obtains the following bibliometric networks: co-author, co-PI (Principal Investigator), documents co-citation, journals co-citation, authors co-citation, bibliographic coupling, author bibliographic coupling and journals bibliographic coupling. Likewise, this tool allows building direct link networks, such as author-references, document-references, journals-references and author-documents networks. In order to represent the networks obtained, different visualization can be obtained: i) Temporal visualization, ii) Geospatial visualization, iii) Choropleth map, iv) Proportional symbol map, v) Topical visualization, and vi) Network visualization. This tool offers a great and adequate number of possibilities in order to represent the different aspects of science.

- VOSviewer: It is a software tool designed for constructing and visualizing bibliometric networks, with journals, researchers, or individual publications as actors, and based on co-citation, bibliographic coupling, or co-authorship relations (Van-Eck; Waltman, 2010). It also offers the possibility of building co-occurrence networks of important terms extracted from a corpus of scientific literature, using a text mining functionality. It was developed by the Centre for Science and Technology Studies (CWTS) at Leiden University (The Netherlands). It can extract bibliographic networks (co-authorship, co-occurrence and citation-based) from bibliographic data. This data is added from files downloaded from WoS, Scopus, Dimensions, PubMed, and RIS format. Moreover, the API of Crossref, Europe PMC and $M A$ can be queried interactively in VOSviewer; if

\section{VOSViewer offers a great visualization and can load and import data from many sources}

we have a set of DOIs, the data can be obtained from the APIs of Semantic Scholar, Open Citations, and WikiData. VOSviewer constructs the map based on a co-occurrence matrix in three steps (Van-Eck; Waltman, 2010): i) Similarity matrix, to apply the VOS mapping technique (Waltman; Van-Eck; Noyons, 2010), using the association strength (VanEck; Waltman, 2007); ii) VOS mapping technique, to construct a map reflecting the similarity measure between items; and iii) Translation, rotation, and reflection, to correct the optimization problem described in the literature ( $\mathbf{O}^{\prime}$ Connell; Borg; Groenen, 1999). About visualization capabilities, this software provides three visualization options: i) network, ii) overlay and iii) density. It is remarkable that the zoom and scroll functionality and smart labelling algorithm to prevent labels overlapping. VOSviewer incorporates the zoom and scroll option in order to facilitate a detailed examination of the map generated. Finally, all the visualizations generated can be saved in different graphical file formats, 


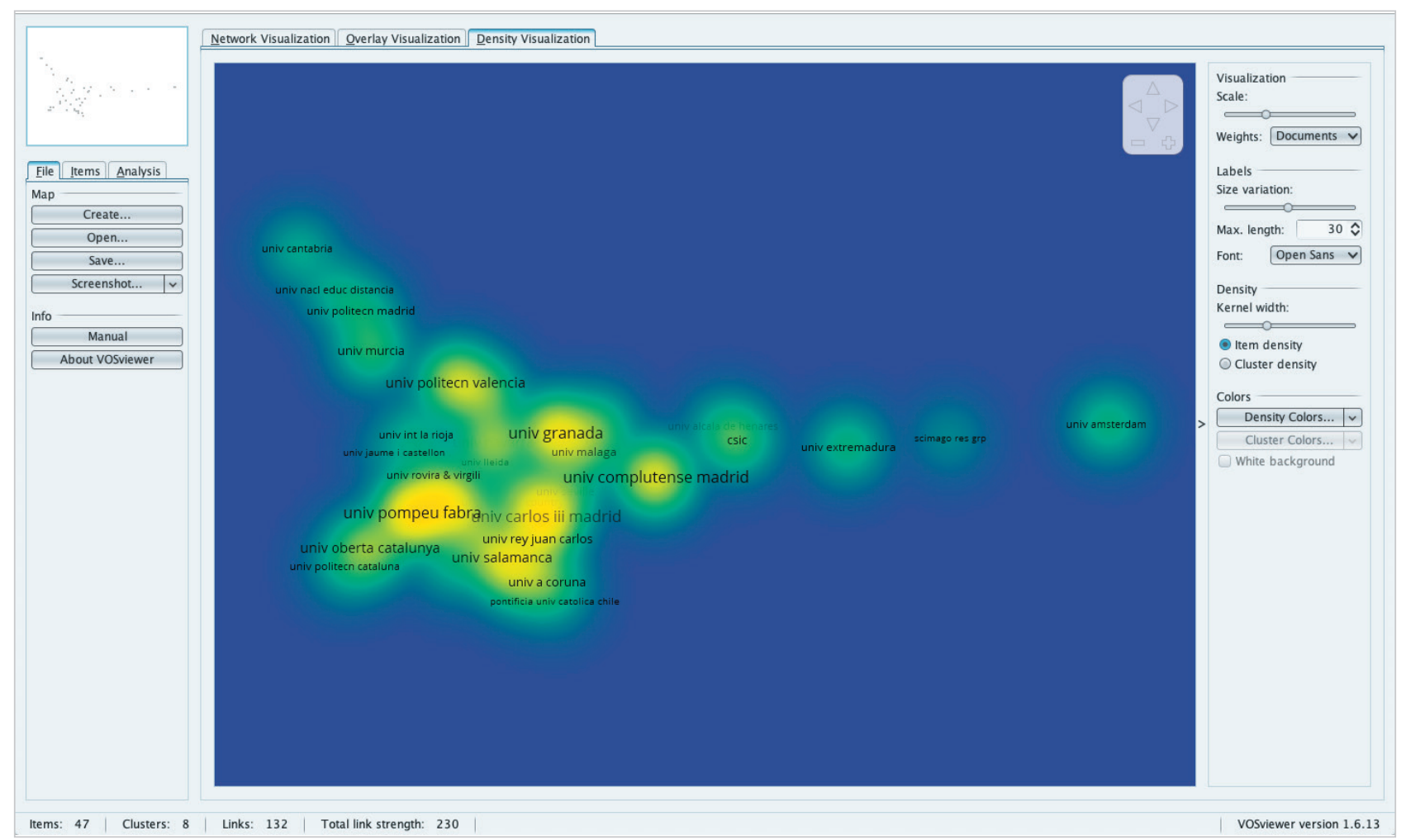

Figure 11. Analysis of the EPI journal with VOSviewer

such as bitmap or vectors. This option makes it easy to include the analysis output in any format, digital or printed. Furthermore, VOSviewer can be launched directly from the web page.

\section{Libraries}

The last category established as tools for bibliometric or scientometric analyses is composed of libraries. This is a concept well-known in the computing area. Computer programs are developed using code, but this code is organized to be used only within this specific software. Conversely, libraries are code that could be used by multiple programs that have no connections to each other. This characteristic makes this kind of tools an interesting option when the user is looking for flexibility and versatility. For example, the analysis of the co-occurrence network could be performed using a library and the visualization is obtained with a different one. Nonetheless, they generally need some knowledge about the language program of the environment that support this code, so it is not a good choice for amateur bibliometricians / scientometricians with any programming knowledge.

Table 4. Characteristics of the libraries

\begin{tabular}{|l|l|l|l|l|}
\hline \multicolumn{1}{|c|}{ Software } & Analyzed version & Year & \multicolumn{1}{c|}{ Developer } & Programming language \\
\hline Bibliometrix & R-3.6.1 & 2019 & University of Naples Federico II & $R$ \\
\hline BiblioTools & 3.2 & 2018 & University of Lyon & Python \\
\hline Citan & $2015.12-1$ & 2015 & Deakin University & $R$ \\
\hline Metaknowledge & - & 2017 & University of Waterloo & Python \\
\hline ScientoPy & 1.4 .0 & 2019 & University of Cauca & Python \\
\hline ScientoText & 0.1 & 2016 & South Asian University & $R$ \\
\hline
\end{tabular}

In that way, a review of the main $R$ and Python libraries is performed in the present manuscript:

- Bibliometrix: It is an open source $R$ package for performing comprehensive SMA (Aria; Cuccurullo, 2017). The stages to perform the analysis are based on de SMA workflow (Cobo; López-Herrera; Herrera-Viedma; Herrera, 2011). As stated above, it empowers the Biblioshiny tool and was developed by Massimo Aria and Corrado Cuccurullo from the University of Naples (Italy). It mainly works with WoS, Scopus and Dimensions data. It incorporates several analysis options and they are subdivided in 7 categories: 1) Overview, 2) Sources, 3) Authors, 4) Documents, 5) Conceptual structures, 6) Intellectual structure, and 7) Social structure. It is a powerful library that can performs complete bibliometric and scientometric analysis. Furthermore, it allows to obtain multiple types of graphs; feature not common in other libraries. 
-BiblioTools: It is a Python library that contains a set of scripts to perform SMA through bibliographic data (Grauwin; Jensen, 2011). As stated in SMA tools section, BiblioTools is the base of the BiblioMaps tools. It is offered as a user-friendly Python tool, since no knowledge about previous programming skills are needed. WoS and Scopus files are used as dataset. The following tasks can be performed using BiblioTools: i) Data acquisition, ii) Data preprocessing, applying parsing and filtering to the raw data, iii) Data analysis, obtaining co-occurrence networks, bibliographic coupling and clusters, iv) Data visualization, the output can be visualized in BiblioMaps or exported to other software, and v) Data report, the analysis obtained can be exported to LaTeX format.

- ClTation Analysis (Citan): This library is a tool pack that allows to perform performance analysis from Scopus data into a SQLite database (Gagolewski, 2011). It works under this kind of database since the user can execute codes to modify the information, such as merge documents or authors, delete duplicates or an specific document or author. Generally, Citan obtain performance analyses through the different actors; it can calculate the $\mathrm{h}$-index, $\mathrm{g}$-index, $\mathrm{r}_{\mathrm{p}}$-index, $\mathrm{I}_{\mathrm{p}}$-index and other general impact indicators. Furthermore, it provides descriptive graphs with the calculated indicators.

- Metaknowledge: It is a Python package for performing bibliometric/scientometric analyses based on performance analysis or SMA (McLevey; Mcllroy-Young, 2017). It mainly works with WoS, PubMed, Scopus and ProQuest Dissertation and Theses data. Concerning the analysis options, it performs longitudinal analysis, standard and multi reference publication year spectroscopy, computational text analysis (such as topic modeling or burst analysis) and network analysis. An interesting feature is the estimating researcher gender; Metaknowledge downloads the Global Name Dataset from Open Gender Tracker's Github repository and matches authors and coauthors' names with the possible gender. Nonetheless, it is not yet a worldwide dataset, so this function has several limitations in global analyses. Moreover, it uses mkD3 package (Bostock; Ogievetsky; Heer, 2011) to visualize the performance and network outputs. Network data can be exported to be visualized in external software.

- ScientoPy: As stated in Section 4, ScientoPy is am open source Python script useful to perform temporal scientometric analysis (Ruiz-Rosero; Ramírez-González; Viveros-Delgado, 2019). It mainly works with WoS and Scopus data. Once the data is extracted, we can perform a preprocessing stage, consisting on document type filtering, author's name normalization, duplicate removal, times cited and document's country and institutions. It allows performing top and specific topics evaluation and wildcard search. Wildcard search is useful to find topics that come in plural and singular or start with an specific root. Furthermore, ScientoPy add some performance indicators to the topics analyzed, such as average growth rate, average document per years and percentage of documents in last years. Several visualization options are allowed, such as time line, horizontal bars, horizontal bars trends, evolution and wordcloud. The graphical oput can be exported to EPS, SVG and PNG formats.

- scientoText: It is a $R$ package to perform some bibliometric/scientometric analysis from bibliographic data based on indicators (Uddin; Bhoosreddy; Tiwari; Singh, 2016). It works with WoS and Scopus data. It is able to obtain different analyses, such as co-authorship matrix, top authors/countries/institutions with their $\mathrm{g}$ and $\mathrm{h}$ indexes and citations, highly cited actors, international collaboration and term frequency. Generally, scientoText offers some bibliometric outputs to characterize an author, institution or country.

\section{Comparative features}

Once the description of the main bibliometric/scientometric tools was carried out, some information about the features of each tool will be addressed. In that way, the features have been subdivided in: a) database sources, b) pre-processing capabilities and c) analysis and visualization options. All the included tools are characterized in Tables 5-7 for a comprehensive understanding.

Table 5 shows the main bibliographic data sources supported by the analyzed tools. It is interesting to remark that all of them incorporate the analysis of WoS and Scopus. Others, such as Bibliometrix / Biblioshiny, CiteSpace and VOSviewer incorporate the analysis of Dimensions, that has expressed its willingness in facilitate the open science. Moreover, Publish or Perish, CiteSpace and VOSviewer include the promising database MA. 
Table 5. Comparative of the databases supported by the reviewed tools

\begin{tabular}{|c|c|c|c|c|c|c|c|c|}
\hline Tools & ڤે & $\frac{n}{\stackrel{\Xi}{2}}$ & 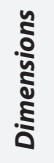 & $\Sigma$ & گ & 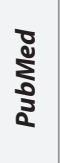 & $\begin{array}{l}\text { ऐั๊ } \\
\text { 仓े }\end{array}$ & Others \\
\hline \multicolumn{9}{|c|}{ General bibliometric and performance analysis } \\
\hline CRExplorer & $x$ & $x$ & & & & & $x$ & \\
\hline Publish or Perish & $x$ & $x$ & & $x$ & $\mathrm{x}$ & & $x$ & \\
\hline ScientoPyUl & $\mathrm{x}$ & $x$ & & & & & & \\
\hline \multicolumn{9}{|c|}{ Science mapping analysis tools } \\
\hline Bibexcel & $\mathrm{x}$ & $x$ & & & & & & ProCite, Bibexcel \\
\hline BiblioShiny & $x$ & $x$ & $\mathrm{x}$ & & & & & \\
\hline BiblioMaps & $x$ & $x$ & & & & & & \\
\hline CiteSpace & $\mathrm{x}$ & $x$ & $x$ & $x$ & & $x$ & $\mathrm{x}$ & ADS, arXiv, CNKI, Cssci, Derwent Patents, NSF, ProQuest, Fulltext, CSV \\
\hline CitNetExplorer & $\mathrm{x}$ & $x$ & & & & & & \\
\hline SCIMAT & $\mathrm{x}$ & $\mathrm{x}$ & & & & & & CSV \\
\hline Sci Tool & $\mathrm{x}$ & $x$ & & & $x$ & & & BibText, EndNote \\
\hline VOSviewer & $\mathrm{x}$ & $x$ & $\mathrm{x}$ & $x$ & & & $\mathrm{x}$ & RIS, PMC, Semantic Scholar, Open Citation, WikiData \\
\hline \multicolumn{9}{|l|}{ Libraries } \\
\hline Bibliometrix & $x$ & $x$ & $\mathrm{x}$ & & & & & \\
\hline BiblioTools & $\mathrm{x}$ & $x$ & & & & & & \\
\hline Citan & & $x$ & & & & & & \\
\hline Metaknowledge & $\mathrm{x}$ & $\mathrm{x}$ & & & & $\mathrm{x}$ & & ProQuest \\
\hline sciento-Text & $x$ & $x$ & & & & & & \\
\hline ScientoPy & $x$ & $x$ & & & & & & \\
\hline
\end{tabular}

Table 6 presents the pre-processing options that the analysed tools incorporate. In that sense, SciMAT is the one offering the widest type of tasks. Almost all the tools include some filters and the second and third features most incorporated are the time slice and the duplicated documents removement. Generally, the pre-processing stage is the unfinished business of the bibliometric/scientometric tools.

Table 6. Comparative of the pre-processing option of the reviewed tools

\begin{tabular}{|c|c|c|c|c|c|c|c|c|}
\hline Tools & $\begin{array}{c}\text { Duplicate } \\
\text { documents }\end{array}$ & $\begin{array}{l}\text { Plurals / } \\
\text { Singulars }\end{array}$ & $\begin{array}{c}\text { String } \\
\text { distance }\end{array}$ & $\begin{array}{c}\text { De-duplica- } \\
\text { tion* }\end{array}$ & Time slice & Stop words & $\begin{array}{c}\text { Data } \\
\text { edition** }\end{array}$ & Filters \\
\hline \multicolumn{9}{|c|}{ General bibliometric and performance analysis } \\
\hline CRExplorer & & & & & & & & $x$ \\
\hline ScientoPyUI & $x$ & & & & & & & $x$ \\
\hline \multicolumn{9}{|c|}{ Science mapping analysis tools } \\
\hline Bibexcel & $x$ & & & & & & & \\
\hline BiblioShiny & & & & & $\mathrm{X}$ & & & $\mathrm{X}$ \\
\hline BiblioMaps & & & & & $\mathrm{X}$ & & & $\mathrm{X}$ \\
\hline CiteSpace & & & & & $x$ & & & $x$ \\
\hline CitNetExplorer & & & & & & & & $\mathrm{X}$ \\
\hline SciMAT & & $\mathrm{X}$ & $x$ & $x$ & $x$ & $x$ & $x$ & $x$ \\
\hline $\mathrm{Sci}^{2} \mathrm{Tool}$ & $x$ & $X$ (stemming) & & & $x$ & $x$ & & $x$ \\
\hline VOSviewer & & & & & & & & $x$ \\
\hline \multicolumn{9}{|l|}{ Libraries } \\
\hline Bibliometrix & & & & & $x$ & & & $x$ \\
\hline BiblioTools & & & & & $x$ & & & $x$ \\
\hline Citan & $x$ & & & $x$ & & & $x$ & \\
\hline Metaknowledge & $x$ & & & & $x$ & & & $x$ \\
\hline ScientoPy & $x$ & & & & & & & $x$ \\
\hline \multicolumn{9}{|c|}{$\begin{array}{l}\text { Some tools were excluded since they do not incorporate any feature. } \\
\text { Excluded: Publish or Perish and scientoText } \\
\text { * The tool allows joining several items (e.g. synonyms, acronyms with full form, authors, etc.) } \\
\text { ** Data can be modified into the tool, so no external software is needed to perform this task. }\end{array}$} \\
\hline
\end{tabular}


Concerning Table 7, the different analysis options of the tools included are specified. Furthermore, a column with the visualization options was added to know how the analysis output can be displayed.

Table 7. Comparative of the analysis and visualization options of the reviewed tools

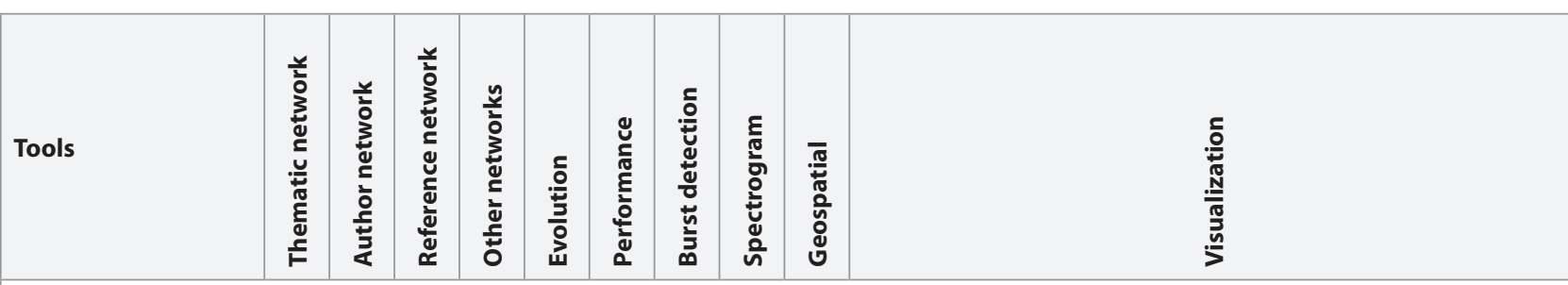

General bibliometric and performance analysis

\begin{tabular}{|l|l|l|l|l|l|l|l|l|l|l}
\hline CRExplorer & & & & & & & & $\mathrm{X}$ & & Spectrogram \\
\hline ScientoPyUI & & & & & $\mathrm{X}$ & $\mathrm{X}$ & & & & Timeline graph, bar graph, evolution graph and word cloud \\
\hline Publish or Perish & & & & & & $\mathrm{X}$ & & & & \\
\hline
\end{tabular}

\section{Science mapping analysis tools}

\begin{tabular}{|l|c|c|c|c|c|c|c|c|c|l|}
\hline Bibexcel & $\mathrm{X}$ & $\mathrm{X}$ & $\mathrm{X}$ & $\mathrm{X}$ & & $\mathrm{X}$ & & & $\mathrm{X}$ & External software \\
\hline BiblioShiny & $\mathrm{X}$ & $\mathrm{X}$ & $\mathrm{X}$ & $\mathrm{X}$ & $\mathrm{X}$ & $\mathrm{X}$ & $\mathrm{X}$ & $\mathrm{X}$ & $\mathrm{X}$ & $\begin{array}{l}\text { Network, three-fields plot, wordcloud, tree map, historiograph, } \\
\text { strategic diagram, evolution map and world map }\end{array}$ \\
\hline BiblioMaps & $\mathrm{X}$ & $\mathrm{X}$ & $\mathrm{X}$ & $\mathrm{X}$ & & $\mathrm{X}$ & & & $\mathrm{X}$ & Network \\
\hline CiteSpace & $\mathrm{X}$ & $\mathrm{X}$ & $\mathrm{X}$ & $\mathrm{X}$ & & $\mathrm{X}$ & $\mathrm{X}$ & & $\mathrm{X}$ & Tree ring, geospatial map \\
\hline CitNetExplorer & & & $\mathrm{X}$ & & & & & & & Network \\
\hline SciMAT & $\mathrm{X}$ & $\mathrm{X}$ & $\mathrm{X}$ & $\mathrm{X}$ & $\mathrm{X}$ & $\mathrm{X}$ & & & & $\begin{array}{l}\text { Strategic diagram, cluster network, overlapping map, evolution } \\
\text { map }\end{array}$ \\
\hline Sci' Tool & $\mathrm{X}$ & $\mathrm{X}$ & $\mathrm{X}$ & $\mathrm{X}$ & & & $\mathrm{X}$ & & $\mathrm{X}$ & Temporal, geospatial map, topical, network \\
\hline VOSviewer & $\mathrm{X}$ & $\mathrm{X}$ & $\mathrm{X}$ & $\mathrm{X}$ & & $\mathrm{X}$ & & & & Network, overlay, density \\
\hline
\end{tabular}

\section{Libraries}

\begin{tabular}{|c|c|c|c|c|c|c|c|c|c|c|}
\hline Bibliometrix & $\mathrm{X}$ & $\mathrm{X}$ & $\mathrm{X}$ & $\mathrm{x}$ & $\mathrm{X}$ & $\mathrm{X}$ & $\mathrm{X}$ & $\mathrm{x}$ & $\mathrm{X}$ & $\begin{array}{l}\text { Network, three-fields plot, wordcloud, tree map, historiograph, } \\
\text { strategic diagram, evolution map and world map }\end{array}$ \\
\hline BiblioTools & $\mathrm{X}$ & $\mathrm{x}$ & $\mathrm{X}$ & $\mathrm{x}$ & & $\mathrm{X}$ & & & $\mathrm{X}$ & Network \\
\hline Citan & & & & & & $\mathrm{x}$ & & & & Bars, box plots and pie chart \\
\hline Metaknowledge & $x$ & $x$ & $x$ & $x$ & & & $x$ & $x$ & & Timeline graph, spectrogram and network \\
\hline scientoText & & $\mathrm{x}$ & & & & $\mathrm{X}$ & & & & \\
\hline ScientoPy & & & & & $\mathrm{x}$ & $\mathrm{x}$ & & & & Timeline graph, bar graph, evolution graph and word cloud \\
\hline
\end{tabular}

\section{Discussion and conclusions}

In this article, a thorough analysis of the most important bibliometric/scientometric tools and software has been developed, highlighting their most important characteristics, and making a global comparative analysis. To do that, the analysis has been focused on four main tools:

a) bibliometric databases (WoS, Scopus, GS, MA and Dimensions),

b) general bibliometric and performance analysis (CRExplorer, ScientoPyUI and Publish or Perish),

c) SMA (Bibexcel, BiblioShiny, BiblioMaps, CiteSpace, CitNetExplorer, SciMAT, Sci2 Tool, VOSviewer), and

d) Python and $R$ libraries (Bibliometrix, BiblioTools, Citan, Metaknowledge, scientoText, ScientoPy).

Although there are different bibliometric databases able to be used as a data source for bibliometric analysis, they have different characteristics and have been developed with a different purpose. One of the most important aspects is the scientific coverage, which has been analyzed and discussed in several studies. Mongeon and Paul-Hus (2016) determined that the Social Sciences and Arts and Humanities are underrepresented in WoS, and the strong influence of English-language is high. Furthermore, although Scopus presents slightly more extensive coverage, it has similar biases than WoS. MA seems to be an alternative for citation analysis, but although it covers life sciences and sciences correctly, engineering, social sciences and humanities are underrepresented in comparison with GS (Harzing; Alakangas, 2017). In that way, according to recent publications (Martín-Martín; Orduña-Malea; Harzing; Delgado-López-Cózar, 2017; Martín-Martín; Orduña-Malea; Delgado-López-Cózar, 2018), GS seems to do a better coverage of some areas of research than WoS (Humanities, Literature \& Arts, Social Sciences, Engineering \& Computer Science, and Economics \& Manage- 
ment) and Scopus (Humanities, Literature \& Arts, and Social Sciences), but it has some drawbacks; such as lack of detailed metadata or difficulty to extract data. To our knowledge, there is no information about the coverage of Dimensions. Then, the data source should be chosen with caution and taking into account the research area where the bibliometric analysis will be performed.

Regarding the exporting options of the databases, there are substantially differences among them. WoS allows to export data in 500 records slots that could be concatenated (500 by 500) with a total limit of 100,000 records per query. It has different export formats, but the most suitable for the bibliometric purpose are plain text or tab-delimited. Scopus allows to download more data in a single petition, but the total records retrieved is limited to the first 2,000 records of the query. Although there are different format options for data exporting, the only one available for bibliometric purpose is the RIS or CSV formats. It is necessary to mention that Scopus allows to download the first 20,000 records of a query in CSV format but limiting the output to only citation data. GS does not bring the possibility of data download, so researcher should employ crawler method (Martín-Martín; Orduña-Malea; Harzing; Delgado-López-Cózar, 2017; Martín-Martín; Orduña-Malea; Delgado-López-Cózar, 2018), or use software tools that integrate the crawling method such as Publish or Perish (see Section 4). The MA search website does not allow data retrieval for bibliometric purpose. However, it offers his data through the Academic Knowledge API or indeed, it allows to access to the different snapshot of the whole dataset through the Open Academic Graph. It should be taken into account that the access to the API is limited to 10,000 requests per month in the free license option. Finally, Dimensions allow retrieving a maximum of 50,000 records per query using a CSV or excel formats. In fact, Dimensions provide a so-called "export for bibliometric mapping", but it is just a specific CSV file.

As was pointed in Section 6, the software tools and libraries were compared according to three groups of features: a) database sources, b) pre-processing options, and c) analysis and visualization options.

Regarding the data sources, almost all the software tools and libraries can import data downloaded from WoS and Scopus, since both databases are the most important and therefore, most of the analyses are based on them. Moreover, Biblioshiny / Bibliometrix, CiteSpace and VOSviewer allow importing data from Dimensions. Publish or Perish, CiteSpace and VOSviewer allow to retrieve data from MA directly from its API. Taking into account GS, Publish or Perish and Sci Tool are the only tools able to work with it. Finally, we should point out that some software tools stand out due to the great number of compatibilities with different data sources. In that sense, we should remark the general bibliometric software tool Publish or Perish, and the SMA software tools CiteSpace and VOSviewer, which are able to work with the most common databases, and also, they allow to work directly with open databases such as Crossref, among others, using their API.

In the bibliometric analysis, the data preprocessing and cleaning is an important and daunting task, that should be performed as the first step. In that sense, the compared software tools and libraries contain a great variety of preprocessing methods and algorithms. First, with the exception of Bibexcel, all the software tools can filter the data in different ways. Additionally, time slicing is allowed by the majority of them (Bibliometrix / Biblioshiny, BiblioMaps / BiblioTool, CiteSpace, SciMAT, Sci ${ }^{2}$ Tool). Duplicate documents removal is also allowed by ScientoPyUI / ScienctoPy, Bibexcel, Sci ${ }^{2}$ Tool, Citan and Metaknowledge. It is important to note that the Publish or Perish and the $R$ library scientoTex do not incorporate any preprocessing method. Finally, regarding the preprocessing capabilities, SciMAT stands out as the most powerful software since it incorporates methods to apply a de-duplication process (manually or using plural / distance, or string distance), time slicing, stop words, and data edition.

We should remark that taking into account the analysis options, Bibliometrix and its user interface Biblioshiny stand out since they incorporate a great variety of different analyses. In practice, since it is recent, most of the analysis developed by the previous software tools have been incorporated in Bibliometrix / Biblioshiby. For instance, it allows to extract and analyze a bibliometric network (thematic, authors and references, among others), performs an evolution analysis, develops a performance analysis based on different indicators, applies a burst detection, draws a spectrogram, and show the geospatial component. On the other hand, taking into account the bibliometric networks, most of the SMA can work with the most common (thematic, authors, and references). Also, Bibexcel, SciMAT and Biblioshiny / Bibliometrix can work with another kind of bibliometric networks. Regarding the $R$ and Python libraries, Metaknowledge can work with a great variety of networks, and also scientoText can work with authors networks. CRExplorer, Bibliometrix / Biblioshiny and metaknowledge can build a spectrogram. The development of an evolution analysis is only available on Bibliometrix / Biblioshiny, SciMAT and ScientoPy / ScientoPyUI. Also, performance analysis (using different measures and indicators) can be developed using the majority of the software tools with the exception of CRExplorer, Sci2 Tool and Metaknowledge. The burst detection is allowed by CiteSpace, Sci2 Tool, Metaknowledge and Bibliometrix / Biblioshiny. Finally, a geospatial analysis could be developed using Bibexcel, Bibliometrix / Biblioshiny, BiblioTools / Bibliomaps, CiteSpace and Sci2 Tool.

As a final conclusion, we should remark that each of the analyzed software tools have their advantages and drawbac$\mathrm{ks}$, and therefore the analyst should choose the adequate software for each specific analysis. At this moment, maybe Bibliometrix and its Shiny platform contain the more extensive set of techniques implemented, and together with the 
easiness of its interface, could be a great software for practitioners. VOSviewer contains a great visualization, and can load and import data with a great number of sources. SciMAT has great preprocessing and exporting capabilities, and also the visualization through the strategic diagram and thematic areas could allow the analyst to focus deeply on some specific research topics.

\section{References}

Alonso, Sergio; Cabrerizo, Francisco-Javier; Herrera-Viedma, Enrique; Herrera, Francisco (2009). "h-index: A review focused in its variants, computation and standardization for different scientific fields". Journal of informetrics, v. 3, n. 4, pp. 273-289.

https://doi.org/10.1016/j.joi.2009.04.001

Alonso, Sergio; Cabrerizo, Francisco-Javier; Herrera-Viedma, Enrique; Herrera, Francisco (2010). "hg-index: A new index to characterize the scientific output of researchers based on the h- and g-indices". Scientometrics, v. 82, n. 2, pp. 391-400.

https://doi.org/10.1007/s11192-009-0047-5

Aria, Massimo; Cuccurullo, Corrado (2017). "Bibliometrix: An R-tool for comprehensive science mapping analysis". Journal of informetrics, v. 11, n. 4, pp. 959-975.

https://doi.org/10.1016/j.joi.2017.08.007

Asimov, Isaac (2010). A short history of chemistry - An introduction to the ideas and concepts of chemistry. New York: Doubleday \& Co.Inc. ISBN: 0313207690

Batagelj, Vladimir; Mrvar, Andrej (2004). "Pajek - Analysis and visualization of large networks". Graph drawing software SE - 4, pp. 77-103. Springer Berlin Heidelberg. ISBN: 9783642622144

https://doi.org/10.1007/978-3-642-18638-7_4

Borgatti, Stephen P.; Everett, Martin G.; Freeman, Linton C. (2002). “Ucinet 6 for Windows: Software for social network analysis". Analytic Technologies, Harvard, MA.

http://www.analytictech.com

Börner, Katy; Chen, Chaomei; Boyack, Kevin W. (2003). "Visualizing knowledge domains". Annual review of information science and technology, v. 37, n. 1, pp. 179-255.

https://doi.org/10.1002/aris.1440370106

Bornmann, Lutz; Haunschild, Robin (2018). "Alternative article-level metrics: The use of alternative metrics in research evaluation". EMBO Reports, v. 19, e47260.

https://doi.org/10.15252/embr.201847260

Bornmann, Lutz; Williams, Richard (2013). "How to calculate the practical significance of citation impact differences? An empirical example from evaluative institutional bibliometrics using adjusted predictions and marginal effects". Journal of informetrics, v. 7, n. 2, pp. 562-574.

https://doi.org/10.1016/j.joi.2013.02.005

Bostock, Michael; Ogievetsky, Vadim; Heer, Jeffrey (2011). “D² Data-driven documents". IEEE Transactions on visualization and computer graphics, v. 17, n. 12, pp. 2301-2309.

https://doi.org/10.1109/TVCG.2011.185

Cabrerizo, Francisco-Javier; Alonso, Sergio; Herrera-Viedma, Enrique; Herrera, Francisco (2010). "q2-Index: Quantitative and qualitative evaluation based on the number and impact of papers in the Hirsch core". Journal of informetrics, v. 4 , n. 1, pp. 23-28.

https://doi.org/10.1016/j.joi.2009.06.005

Chapman, Karen; Ellinger, Alexander E. (2019). "An evaluation of Web of Science, Scopus and Google Scholar citations in operations management". International journal of logistics management, v. 30, n. 4, pp. 1039-1053.

https://doi.org/10.1108/IJLM-04-2019-0110

Chen, Chaomei (2006). "CiteSpace II: Detecting and visualizing emerging trends and transient patterns in scientific literature". Journal of the American Society for Information Science and Technology, v. 57, pp. 359-377.

https://doi.org/10.1002/asi.20317

Chen, Chaomei (2017). "Science mapping: A systematic review of the literature". Journal of data and information science, v. 2, n. 2, pp. 1-40.

https://doi.org/10.1515/jdis-2017-0006

Chen, Chaomei (2019). How to use CiteSpace. Victoria: Leanpub. 
Cobo, Manuel J.; López-Herrera, Antonio G.; Herrera-Viedma, Enrique; Herrera, Francisco (2011a). "Science mapping software tools: Review, analysis, and cooperative study among tools". Journal of the American Society for Information Science and Technology, v. 62, n. 7, pp. 1382-1402.

https://doi.org/10.1002/asi.21525

Cobo, Manuel J.; López-Herrera, Antonio G.; Herrera-Viedma, Enrique; Herrera, Francisco (2011b). “An approach for detecting, quantifying, and visualizing the evolution of a research field: A practical application to the fuzzy sets theory field". Journal of informetrics, v. 5, n. 1, pp. 146-166.

https://doi.org/10.1016/j.joi.2010.10.002

Cobo, Manuel J.; López-Herrera, Antonio G.; Herrera-Viedma, Enrique; Herrera, Francisco (2012). "SciMAT: A new science mapping analysis software tool". Journal of the American Society for Information Science and Technology, v. 63, n. 8, pp. 1609-1630.

https://doi.org/10.1002/asi.22688

Egghe, Leo (2006). "Theory and practise of the g-index". Scientometrics, v. 69, pp. 131-152.

https://doi.org/10.1007/s11192-006-0144-7

Ellegaard, Ole; Wallin, Johan A. (2015). "The bibliometric analysis of scholarly production: How great is the impact?" Scientometrics, v. 105, pp. 1809-1831.

https://doi.org/10.1007/s11192-015-1645-z

Fabregat-Aibar, Laura; Barberà-Mariné, M. Glòria; Terceño, Antonio; Pié, Laia (2019). “A bibliometric and visualization analysis of socially responsible funds". Sustainability, v. 11, n. 9.

https://doi.org/10.3390/su11092526

Gagolewski, Marek (2011). "Bibliometric impact assessment with R and the Citan package”. Journal of informetrics, v. 5, n. 4, pp. 678-692.

https://doi.org/10.1016/j.joi.2011.06.006

Garfield, Eugene; Pudovkin, Alexander I.; Istomin, Vladimir S. (2003). "Why do we need algorithmic historiography?" Journal of the American Society for Information Science and Technology, v. 54, n. 5, pp. 400-412.

https://doi.org/10.1002/asi.10226

Glänzel, Wolfgang (2012). "Bibliometric methods for detecting and analysing emerging research topics". El profesional de la información, v. 21, n. 2, pp. 194-201.

https://doi.org/10.3145/epi.2012.mar.11

Grauwin, Sebastian; Jensen, Pablo (2011). “Mapping scientific institutions”. Scientometrics, v. 89, article 943. https://doi.org/10.1007/s11192-011-0482-y

Gusenbauer, Michael (2019). “Google Scholar to overshadow them all? Comparing the sizes of 12 academic search engines and bibliographic databases". Scientometrics, v. 118, pp. 177-214.

https://doi.org/10.1007/s11192-018-2958-5

Gutiérrez-Salcedo, María; Martínez, M. Ángeles; Moral-Muñoz, José A.; Herrera-Viedma, Enrique; Cobo, Manuel J. (2018). "Some bibliometric procedures for analyzing and evaluating research fields". Applied intelligence, v. 48, n. 5, pp. 1275-1287.

https://doi.org/10.1007/s10489-017-1105-y

Harzing, Anne-Wil (2008). "Reflections on the h-index".

https://harzing.com/publications/white-papers/reflections-on-the-h-index

Harzing, Anne-Wil; Alakangas, Satu (2017). “Microsoft Academic: is the phoenix getting wings?” Scientometrics, v. 110, n. 1, pp. 371-383.

https://doi.org/10.1007/s11192-016-2185-x

Harzing, Anne-Will K.; Van-der-Wal, Ron (2008). “Google Scholar as a new source for citation analysis". Ethics in science and environmental politics, v. 8, n. 1, pp. 61-73.

https://doi.org/10.3354/esep00076

Haunschild, Robin; Hug, Sven E.; Brändle, Martin P.; Bornmann, Lutz (2018). "The number of linked references of publications in Microsoft Academic in comparison with the Web of Science". Scientometrics, v. 114, pp. 367-370.

https://doi.org/10.1007/s11192-017-2567-8

Hirsch, Jorge E. (2005). "An index to quantify an individual's scientific research output". Proceedings of the National Academy of Sciences of the United States of America, v. 102, n. 46, pp. 16569-16572.

https://doi.org/10.1073/pnas.0507655102 
Hug, Sven E.; Ochsner, Michael; Brändle, Martin P. (2017). "Citation analysis with Microsoft Academic". Scientometrics, v. 111, pp. 371-378. https://doi.org/10.1007/s11192-017-2247-8

Ibba, Simona; Pani, Filippo-Eros; Stockton, John-Gregory; Barabino, Giulio; Marchesi, Michele; Tigano, Danilo (2017). "Incidence of predatory journals in computer science literature". Library review, v. 66, n. 6/7, pp. 505-522.

https://doi.org/10.1108/LR-12-2016-0108

Martín-Martín, Alberto; Orduña-Malea, Enrique; Delgado-López-Cózar, Emilio (2018). “Coverage of highly-cited documents in Google Scholar, Web of Science, and Scopus: a multidisciplinary comparison". Scientometrics, v. 116, pp. 2175-2188.

https://doi.org/10.1007/s11192-018-2820-9

Martín-Martín, Alberto; Orduña-Malea, Enrique; Harzing, Anne-Wil; Delgado-López-Cózar, Emilio (2017). “Can we use Google Scholar to identify highly-cited documents?". Journal of informetrics, v. 11, n. 1, pp. 152-163.

https://doi.org/10.1016/j.joi.2016.11.008

Marx, Werner; Bornmann, Lutz; Barth, Andreas; Leydesdorff, Loet (2014). "Detecting the historical roots of research fields by reference publication year spectroscopy (RPYS)". Journal of the Association for Information Science and Technology, v. 65, n. 4, pp. 751-764.

https://doi.org/10.1002/asi.23089

McLevey, John; Mcllroy-Young, Reid (2017). “Introducing metaknowledge: Software for computational research in information science, network analysis, and science of science". Journal of informetrics, v. 11, n. 1, pp. 176-197.

https://doi.org/10.1016/j.joi.2016.12.005

Mongeon, Philippe; Paul-Hus, Adèle (2016). "The journal coverage of Web of Science and Scopus: a comparative analysis". Scientometrics, v. 106, pp. 213-228.

https://doi.org/10.1007/s11192-015-1765-5

Moral-Muñoz, José A.; López-Herrera, Antonio G.; Herrera-Viedma, Enrique; Cobo, Manuel J. (2019). "Science mapping analysis software tools: A review". Springer handbook of science and technology indicators, pp. 159-185.

https://doi.org/10.1007/978-3-030-02511-3_7

Narin, Francis; Hamilton, Kimberly S. (1996). “Bibliometric performance measures”. Scientometrics, v. 36, pp. 293-310. https://doi.org/10.1007/BF02129596

Noyons, Ed C. M.; Moed, Henk F.; Van-Raan, Anthony F. J. (1999). “Integrating research performance analysis and science mapping". Scientometrics, v. 46, pp. 591-604.

https://doi.org/10.1007/BF02459614

O'Connell, Ann A.; Borg, Ingwer; Groenen, Patrick (1999). "Modern multidimensional scaling: Theory and applications". Journal of the American Statistical Association, v. 94, n. 445, pp. 338-339.

https://doi.org/10.2307/2669710

Orduña-Malea, Enrique; Delgado-López-Cózar, Emilio (2018). “Dimensions: re-discovering the ecosystem of scientific information". El profesional de la información, v. 27, n. 2, pp. 420-431.

https://doi.org/10.3145/epi.2018.mar.21

Pan, Xuelian; Yan, Erjia; Cui, Ming; Hua, Weina (2018). "Examining the usage, citation, and diffusion patterns of bibliometric mapping software: A comparative study of three tools". Journal of informetrics, v. 12, n. 2, pp. $481-493$. https://doi.org/10.1016/j.joi.2018.03.005

Persson, Olle; Danell, Rickard; Wiborg-Schneider, Jesper (2009). "How to use Bibexcel for various types of bibliometric analysis". Celebrating scholarly communications studies: A festschrift for Olle Persson at his 60 ${ }^{\text {th }}$ birthday, pp. 9-24. https://portal.research.lu.se/ws/files/5902071/1458992.pdf

Pradhan, Pallab (2016). "Science mapping and visualization tools used in bibliometric \& scientometric studies: An overview". Inflibnet newsletter.

http://hdl.handle.net/1944/2132

Pritchard, Alan (1969). "Statistical bibliography or bibliometrics?". Journal of documentation, v. 25, n. 4, pp. 348-349.

Ruiz-Rosero, Juan; Ramírez-González, Gustavo; Viveros-Delgado, Jesús (2019). "Software survey: ScientoPy, a scientometric tool for topics trend analysis in scientific publications". Scientometrics, v. 121, n. 2, pp. 1165-1188.

https://doi.org/10.1007/s11192-019-03213-w 
Sangam, Shivappa L.; Mogali, Shivaranjini S. (2012). "Mapping and visualization softwares tools: a review". Content management in networked environment, p. 11.

https://www.researchgate.net/publication/260165605_Mapping_and_Visualization_Softwares_tools_a_review

Sci2 Team (2009). "Science of science (Sci2) Tool".

http://sci.slis.indiana.edu

Skute, Igors; Zalewska-Kurek, Kasia; Hatak, Isabella; De-Weerd-Nederhof, Petra (2019). “Mapping the field: a bibliometric analysis of the literature on university-industry collaborations". Journal of technology transfer, v. 44, n. 3, pp. 916-947.

https://doi.org/10.1007/s10961-017-9637-1

Small, Henry (1999). "Visualizing science by citation mapping". Journal of the American Society for Information Science, v. 50, n. 9, pp. 799-813.

https://doi.org/10.1002/(SICI)1097-4571(1999)50:9<799::AID-ASI9>3.0.CO;2-G

Thelwall, Mike (2018). “Dimensions: A competitor to Scopus and the Web of Science?". Journal of informetrics, v. 12, n. 2, pp. 430-435.

https://doi.org/10.1016/j.joi.2018.03.006

Thor, Andreas; Marx, Werner; Leydesdorff, Loet; Bornmann, Lutz (2016). “Introducing CitedReferencesExplorer (CRExplorer): A program for reference publication year spectroscopy with cited references standardization". Journal of Informetrics, v. 10, n. 2, pp. 503-515.

https://doi.org/10.1016/j.joi.2016.02.005

Uddin, Ashraf; Bhoosreddy, Jaideep; Tiwari, Marisha; Singh, Vivek-Kumar (2016). "A Sciento-text framework to characterize research strength of institutions at fine-grained thematic area level". Scientometrics, v. 106, n. 3, pp. 1135-1150. https://doi.org/10.1007/s11192-016-1836-2

Van-Eck, Nees-Jan; Waltman, Ludo (2007). "Bibliometric mapping of the computational intelligence field". International journal of uncertainty, fuzziness and knowlege-based systems, v. 15, n. 5, pp. 625-645.

https://doi.org/10.1142/S0218488507004911

Van-Eck, Nees-Jan; Waltman, Ludo (2010). "Software survey: VOSviewer, a computer program for bibliometric mapping". Scientometrics, v. 84, n. 2, pp. 523-538.

https://doi.org/10.1007/s11192-009-0146-3

Van-Eck, Nees-Jan; Waltman, Ludo (2014). "CitNetExplorer: A new software tool for analyzing and visualizing citation networks". Journal of informetrics, v. 8, n. 4, pp. 802-823.

https://doi.org/10.1016/j.joi.2014.07.006

Van-Raan, Anthony F. J. (1999). "Advanced bibliometric methods for the evaluation of universities". Scientometrics, v. 45, n. 3, pp. 417-423.

https://doi.org/10.1007/BF02457601

Van-Raan, Anthony F. J. (2004a). “Measuring science. Capita selecta of current main issues”. In: Moed, Henk F.; Glänzel, Wolfgang; Schmoch, Ulrich (eds.). Handbook of quantitative science and technology research: The use of publication and patent statistics in studies of s\&t systems. Wolters Kluwer, pp. 19-50. ISBN: 1402027028

Van-Raan, Anthony F. J. (2004b). "Sleeping beauties in science". Scientometrics, v. 59, n. 3, pp. 467-472. https://doi.org/10.1023/B:SCIE.0000018543.82441.f1

Veeranjaneyulu, K. (2017). "Altmetrics: new tools to measure research impact in the digitally networked". In: National conference of agricultural librarians and user community.

https://bit.ly/2rS7e1e

Waltman, Ludo; Van-Eck, Nees-Jan; Noyons, Ed C. M. (2010). “A unified approach to mapping and clustering of bibliometric networks". Journal of Informetrics, v. 4, n. 4, pp. 629-635.

https://doi.org/10.1016/j.joi.2010.07.002 